SLAC-PUB-8324

UCLA/99/TEP/48

Saclay-SPhT-T99/147

hep-ph/0001001

January 1, 2000

\title{
A Two-Loop Four-Gluon Helicity Amplitude in QCD
}

\author{
Z. Bern ${ }^{\star}$ \\ Department of Physics and Astronomy \\ UCLA, Los Angeles, CA 90095-1547 \\ L. Dixon ${ }^{\dagger}$ \\ Stanford Linear Accelerator Center \\ Stanford University \\ Stanford, CA 94309 \\ and \\ D.A. Kosower \\ Service de Physique Théorique \\ Centre d'Etudes de Saclay \\ F-91191 Gif-sur-Yvette cedex, France
}

\begin{abstract}
We present the two-loop pure gauge contribution to the gluon-gluon scattering amplitude with maximal helicity violation. Our construction of the amplitude does not rely directly on Feynman diagrams, but instead uses its analytic properties in $4-2 \epsilon$ dimensions. We evaluate the loop integrals appearing in the amplitude through $\mathcal{O}\left(\epsilon^{0}\right)$ in terms of polylogarithms.
\end{abstract}

Submitted to JHEP

${ }^{\star}$ Research supported by the US Department of Energy under grant DE-FG03-91ER40662.

${ }^{\dagger}$ Research supported by the US Department of Energy under grant DE-AC03-76SF00515.

\#Laboratory of the Direction des Sciences de la Matière of the Commissariat à l'Energie Atomique of France. 


\section{Introduction}

The quest for more precise theoretical predictions for particle production at colliders requires calculations to ever-higher orders in the perturbative expansion of field theories. At tree level and at one loop, workers have made substantial progress in recent years in computing multi-parton QCD scattering amplitudes $[1,2]$. Several important quantities, such as the total cross section for $e^{+} e^{-}$ annihilation into hadrons and the QCD $\beta$-function, have been calculated up to four loops [3]. In contrast, essentially no higher-loop fixed-order results are available for multi-parton processes depending on more than one kinematic variable. In particular, the two-loop amplitudes necessary for reducing the theoretical uncertainty in the measurement of $\alpha_{s}$ from $e^{+} e^{-}$jet rates and other event-shape variables remain uncalculated.

There has, however, been some progress in developing general formalisms for the computation of next-to-next-to-leading-order (NNLO) jet cross sections in QCD. This includes the structure of the infrared singularities that arise when partons are unresolved [4], and the explicit evaluation of two-loop integrals $[5,6]$ associated with massless processes. In the special case of maximal $N=4$ supersymmetry, one of the authors and his collaborators have computed the two-loop four-point amplitudes in terms of scalar double box integrals [7].

In this paper we compute a non-supersymmetric two-loop QCD amplitude. Our method for obtaining the amplitude is based on using unitarity to determine its functional form. This program has been carried out for many one-loop and a small of number of two-loop amplitudes [8, 7, 9, 10]. The latter computations were for the special case of theories with $N=4$ or $N=8$ supersymmetry. The two-loop amplitude considered here is more difficult to obtain because one cannot directly rely on supersymmetry. We will have to evaluate the unitarity cuts with more care, by working in dimensional regularization with arbitrary dimension $D=4-2 \epsilon$, taking the limit $\epsilon \rightarrow 0$ only at the end.

The particular process we study is $g^{-} g^{-} \rightarrow g^{+} g^{+}$in pure gauge theory, where $g^{-}\left(g^{+}\right)$denotes a negative (positive) helicity gluon. In a helicity convention in which all gluons are treated as outgoing, the amplitude is that for four identical-helicity gluons, $0 \rightarrow g^{+} g^{+} g^{+} g^{+}$. This helicity amplitude vanishes at tree level [11], and consequently the two-loop term we compute here contributes to the cross section only at one order beyond NNLO. Nevertheless, the techniques used here should be applicable to the helicity configurations that do contribute at NNLO.

This paper is organized as follows. In section 2 we give an overview of the unitarity technique as applied to two-loop amplitudes. Section 3 describes the construction of some useful auxiliary amplitudes where scalars replace some of the gluons circulating in the loops. The full two-loop four-gluon amplitude is presented in section 4 . In section 5 we verify that this amplitude has the combined ultraviolet and infrared divergence structure expected on general grounds [12, 13]. An appendix contains the expansion in $\epsilon$, through $\mathcal{O}\left(\epsilon^{0}\right)$, of the two-loop integrals appearing in the amplitude, expressed in terms of standard functions (polylogarithms). 


\section{Overview of method}

We reconstruct the two-loop four-gluon amplitude from its unitarity cuts, by evaluating the latter in dimensional regularization [14] with $D=4-2 \epsilon$. Traditional applications of unitarity, via dispersion relations, suffer in general from subtraction ambiguities. These ambiguities are related to the appearance of rational functions with vanishing imaginary parts, $R\left(S_{i}\right)$, where $S_{i}=\{s, t, u, \ldots\}$ are the kinematic variables for the amplitude. However, dimensionally-regulated amplitudes for massless particles necessarily acquire a factor of $\left(-S_{i}\right)^{-\epsilon}$ for each loop, from the measure $\int d^{D} L$ and dimensional analysis. For small $\epsilon,\left(-S_{i}\right)^{-\epsilon} R\left(S_{i}\right)=R\left(S_{i}\right)-\epsilon \ln \left(-S_{i}\right) R\left(S_{i}\right)+\mathcal{O}\left(\epsilon^{2}\right)$, so every term has an imaginary part (for some $S_{i}>0$ ), though not necessarily in those terms which survive as $\epsilon \rightarrow 0$. Thus, the unitarity cuts evaluated to $\mathcal{O}(\epsilon)$ provide sufficient information for the complete reconstruction of an amplitude through $\mathcal{O}\left(\epsilon^{0}\right)$, subject only to the usual prescription dependence associated with renormalization $[8,2]$. The subtraction ambiguities that arise in traditional dispersion relations are related to the non-convergence of dispersion integrals. A dimensional regulator makes such integrals well-defined and correspondingly eliminates the subtraction ambiguities. In a sense, we use dimensional regularization as a calculational tool, beyond its usual role as an infrared and ultraviolet regulator.

We make use of the fact that the amplitudes we are computing are also expressible in terms of Feynman diagrams. They can therefore be expressed as a sum of loop integrals multiplied by kinematic coefficients. We can therefore bypass the dispersion integrals by identifying the loop integrals whose cuts match the appropriate products of tree or one-loop amplitudes. Our main task will then be to compute the integrands of the two-loop integrals from the tree or one-loop amplitudes. We obtain the full amplitude in terms of various loop integrals, by requiring consistency of the different cuts. The consistency conditions are relatively simple to implement because they hold before integrating over intermediate-state momenta. Because no integration is required, we can verify the resulting form numerically (to high accuracy), even when analytic simplification of some of the cuts is impractical. This approach is powerful because amplitudes on both sides of the cuts can be simplified, including cancellations dictated by gauge invariance, before their products are computed. This reduces greatly the number of terms in intermediate steps. The final results are quite compact when expressed in terms of loop-momentum integrals, thus simplifying the task of performing the integrals explicitly. The cut-based method thereby provides a short-cut to final expressions for amplitudes.

Let us begin by considering the two-particle cuts of the full amplitude, including color factors. In the $s$ channel, in general, there are two contributions to such cuts, as displayed in fig. 1 . These are,

$$
\begin{aligned}
&\left.\mathcal{A}_{4}^{2 \text {-loop }}(1,2,3,4)\right|_{2 \text {-cut }} \\
& \quad=\sum_{\substack{\text { physical } \\
\text { states }}}\left[\mathcal{A}_{4}^{\text {tree }}\left(1,2,-\ell_{2},-\ell_{1}\right) \times \mathcal{A}_{4}^{1-\text { loop }}\left(\ell_{1}, \ell_{2}, 3,4\right)+\mathcal{A}_{4}^{1 \text {-loop }}\left(1,2,-\ell_{2},-\ell_{1}\right) \times \mathcal{A}_{4}^{\text {tree }}\left(\ell_{1}, \ell_{2}, 3,4\right)\right],
\end{aligned}
$$

where the notation '2-cut' means cutting the lines corresponding to $\ell_{1,2}$, taking the absorptive part, 
and extracting the integrand of the resulting phase-space integral. On the right-hand side the legs labeled $\ell_{1,2}$ are thus on shell in $D$ dimensions, $\ell_{i}^{2}=0$. Here $\mathcal{A}_{n}^{\text {tree }}, \mathcal{A}_{n}^{1 \text {-loop }}$ and $\mathcal{A}_{n}^{2 \text {-loop }}$ are full $n$-point amplitudes, including all color factors. For each external leg we have abbreviated the dependence on the outgoing external momenta $k_{i}$, color indices $a_{i}$, and polarizations $\varepsilon_{i}$, by the label $i$. The sum over physical states crossing the cut contains a sum over colors. If the cut leg is a gluon, its associated polarization vector $\varepsilon_{\ell_{i}}$ must be transverse, $\varepsilon_{\ell_{i}} \cdot \ell_{i}=0$. In the sum over states, this can be imposed by a transverse projector $P_{\mu \nu}$ acting on the Lorentz indices crossing the cut.

We must also consider three-particle cuts. In the $s$-channel this cut is displayed in fig. 2 , and is given in terms of five-point tree amplitudes by,

$$
\left.\mathcal{A}_{4}^{2 \text {-loop }}(1,2,3,4)\right|_{3 \text {-cut }}=\sum_{\substack{\text { physical } \\ \text { states }}} \mathcal{A}_{5}^{\text {tree }}\left(1,2,-\ell_{3},-\ell_{2},-\ell_{1}\right) \times \mathcal{A}_{5}^{\text {tree }}\left(\ell_{1}, \ell_{2}, \ell_{3}, 3,4\right),
$$

where the notation ' 3 -cut' means an operation analogous to that denoted by '2-cut', but cutting the three lines corresponding to $\ell_{1,2,3}$. On the right-hand side the corresponding legs are on shell. The two-loop amplitude will satisfy both eqs. (2.1) and (2.2). These $s$-channel cuts, together with the similar $t$ - and $u$-channel ones, provide the analytic information necessary to obtain the complete fourpoint two-loop amplitudes. (The invariants $s, t, u$ are the usual Mandelstam variables, $s=\left(k_{1}+k_{2}\right)^{2}$, $t=\left(k_{1}+k_{4}\right)^{2}$, and $u=\left(k_{1}+k_{3}\right)^{2}$.)
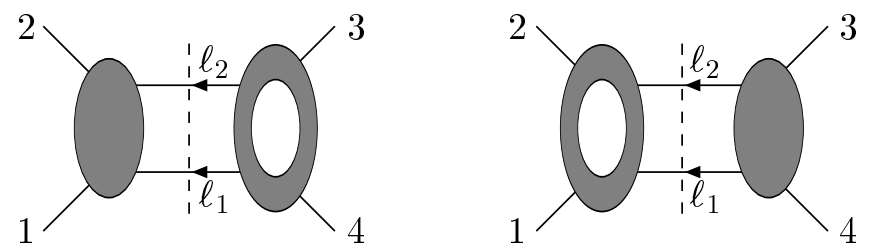

Figure 1: The $s$-channel two-particle cuts of a two-loop amplitude as products of tree and one-loop amplitudes. We take all external momenta to be outgoing. The dashed lines represent the cuts.

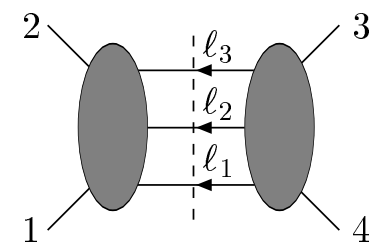

Figure 2: The three-particle cut of a two-loop amplitude.

The unitarity-based technique may also be viewed as an alternate way of evaluating sets of ordinary Feynman diagrams. It does this by collecting together gauge-invariant sets of terms which correspond to the residues of poles in the integrands. The poles are those of the propagators of the cut lines. This corresponds to a region of loop-momentum integration where the cut loop momenta go on shell and the corresponding internal lines become the intermediate states in a unitarity relation. From this point of view, we may consider even more restricted regions of loop momentum integration, 
where additional internal lines go on shell (and, if they are gluons, become transverse as well). This amounts to imposing cut conditions on additional internal lines.

In this vein it is useful for us to define a 'double' two-particle generalized cut for a two-loop fourpoint amplitude. This quantity, illustrated in fig. 3 , is written in terms of on-shell tree amplitudes as

$$
\left.\mathcal{A}_{4}^{2 \text {-loop }}(1,2,3,4)\right|_{2 \times 2 \text {-cut }}=\sum_{\substack{\text { physical } \\ \text { states }}} \mathcal{A}_{4}^{\text {tree }}\left(1,2,-\ell_{2},-\ell_{1}\right) \times \mathcal{A}_{4}^{\text {tree }}\left(\ell_{1}, \ell_{2},-\ell_{3},-\ell_{4}\right) \times \mathcal{A}_{4}^{\text {tree }}\left(\ell_{4}, \ell_{3}, 3,4\right),
$$

where the on-shell conditions are again imposed on the $\ell_{i}, i=1,2,3,4$ appearing on the right-hand side. This equation should not be interpreted as trying to take 'the imaginary part of an imaginary part'. Rather it should be understood in the sense of the previous paragraph as supplying information about the integrand of the two-loop amplitude. It supplies only part of the information contained in the usual two-particle cut (2.1), which effectively imposes only two kinematic constraints on the intermediate lines. However, it is simpler to evaluate because it is composed only of tree amplitudes. Its computation is thus a natural first step. As we shall see this double cut contains 'most' of the final result for the amplitude we are computing in this paper.

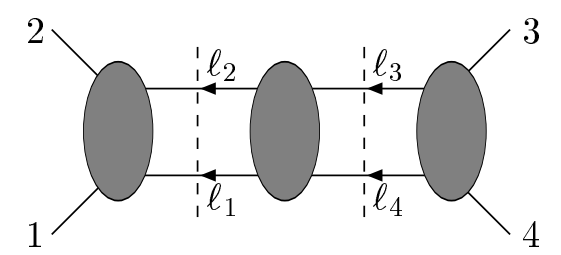

Figure 3: The s-channel double two-particle cut of a two-loop amplitude separates it into a product of three tree amplitudes. The dashed lines represent the generalized cuts.

Consider the two-loop four-point amplitude with all particles in the adjoint representation of the gauge group $S U\left(N_{c}\right)$. We may write its color decomposition as follows [7],

$$
\begin{array}{r}
\mathcal{A}_{4}^{2-\text { loop }}(1,2,3,4)=g^{6} \sum_{\sigma \in S_{4} / Z_{4}} N_{c}^{2} \operatorname{Tr}\left[T^{a_{\sigma(1)}} T^{a_{\sigma(2)}} T^{a_{\sigma(3)}} T^{a_{\sigma(4)}}\right]\left[A_{4 ; 1,1}^{\mathrm{LC}}(\sigma(1), \sigma(2), \sigma(3), \sigma(4))\right. \\
\left.+\frac{1}{N_{c}^{2}} A_{4 ; 1,1}^{\mathrm{SC}}(\sigma(1), \sigma(2), \sigma(3), \sigma(4))\right] \\
+g^{6} \sum_{\sigma \in S_{4} / Z_{2}^{3}} N_{c} \operatorname{Tr}\left[T^{a_{\sigma(1)}} T^{a_{\sigma(2)}}\right] \operatorname{Tr}\left[T^{a_{\sigma(3)}} T^{a_{\sigma(4)}}\right] A_{4 ; 1,3}(\sigma(1), \sigma(2) ; \sigma(3), \sigma(4)),
\end{array}
$$

where $A_{4 ; 1,1}$ and $A_{4 ; 1,3}$ are 'partial amplitudes'. (Our fundamental representation color matrices are normalized by $\operatorname{Tr}\left[T^{a} T^{b}\right]=\delta^{a b}$.) The notation ' $S_{4} / Z_{4}$ ' denotes the set of all permutations of four objects $S_{4}$, omitting the cyclic transformations. The notation ' $S_{4} / Z_{2}^{3}$ ' refers again to the set of permutations of four objects, omitting those permutations which exchange labels within a single trace or exchange the two traces. That is, $S_{4} / Z_{2}^{3}=\{(1234),(1324),(1423)\}$. There is a similar decomposition for contributions linearly proportional to the number of particles in the fundamental representation, but with each term lacking one factor of $N_{c}$ (see eq. (3.1) below). 
These color decompositions have the virtue of making manifest the gauge invariance of the partial amplitudes. In addition the computation of the leading-color partial amplitudes from their cuts is straightforward. However, the cut structure of the subleading-color partial amplitudes is more complicated. For this reason it will prove convenient to use a somewhat different color decomposition, based on structure constants $f^{a b c}$ and specialized to the all-plus helicity amplitude, whose specification we postpone until section 4 .

In evaluating the cuts we use the 't Hooft-Veltman (HV) variant of dimensional regularization [14]. (For a discussion of variants of dimensional regularization see refs. [15, 16] and refs. [17, 18]. Note however, that the one-loop near-equivalence of the four-dimensional helicity scheme and of dimensional reduction assumed in refs. [17, 18] may not hold at two loops.) In this scheme observed external polarization vectors and momenta are four-dimensional, while unobserved and internal ones are $D$-dimensional. Cut lines are considered 'internal'. We use the convention that $D>4(\epsilon<0)$ so that a four-dimensional vector is given by setting the $(-2 \epsilon)$-dimensional components to zero. The $\mathrm{HV}$ variant violates supersymmetry Ward identities, but is commonly used and is straightforward to implement.

In sewing the cut gluon lines we use the transverse, or physical state, projector,

$$
P_{\mu \nu}(\ell, r)=-\eta_{\mu \nu}+\frac{\ell_{\mu} r_{\nu}+r_{\mu} \ell_{\nu}}{r \cdot \ell},
$$

where $\ell$ is the gluon momentum and $r$ is an arbitrary null 'reference' momentum that drops out of all final expressions. The Lorentz indices in this expression are all $D$-dimensional, but for convenience we will take $r$ to lie in the four-dimensional subspace. For cases where only a single gluon crosses the cut, the second term in the projector drops out since it is contracted with conserved currents; for cases where two or more gluons cross the cut, the second term ensures that unphysical states do not circulate in the loops. We denote the number of gluon states circulating in the loop by $D_{s}-2$, which is implemented by the Minkowski metric sum $\eta^{\mu \nu} \eta_{\mu \nu}=D_{s}$. In the HV scheme [14] which we are using here, $D_{s}=D$ should be taken in the final expressions. However, it is useful to keep $D_{s}$ and $D$ independent in intermediate steps.

\section{Two-loop amplitudes with an internal scalar loop}

Although the double cut (2.3) contains only tree amplitudes, the gluons crossing a generalized cut carry Lorentz indices along with them, making the algebra still non-trivial. Contributions with scalars crossing the cut are easier to compute first, and provide useful information about the gluon amplitude. Indeed at one loop, the contributions to identical-helicity all-gluon amplitudes with a scalar circulating in the loop are precisely equal, up to a trivial overall constant, to the contributions with either a fermion or gluon in the loop [19]. This may be understood in terms of supersymmetry Ward identities [11]: $\mathcal{A}_{n}(+,+, \ldots,+)$ vanishes to any loop order in a (supersymmetrically regulated) supersymmetric theory, and the combinations of gluon + fermion and scalar + fermion circulating in a single loop are supersymmetric.

At two loops, even were we to use a manifestly supersymmetric regulator, we should not expect the simple one-loop proportionality between scalar and gluon loop contributions to persist. Nonethe- 
less, it is plausible that contributions from different internal particles will be closely related. Indeed, for the all-plus helicity amplitude, we have found it useful to evaluate first the contribution with an internal scalar circulating around one of the loops. It serves as an excellent guide to an ansatz for the pure gluon case.

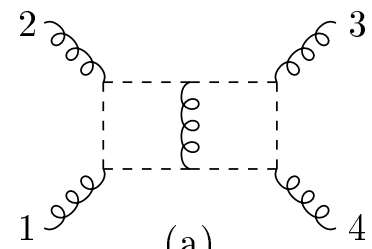

(a)

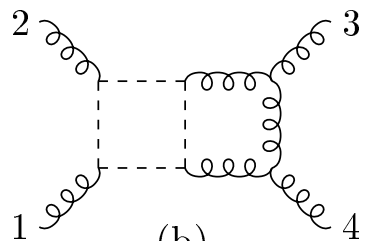

(b)

Figure 4: Representative diagrams for the contributions with a single scalar loop. The class (a) diagrams can be drawn on the plane with all external legs on the outside, and one internal gluon line on the inside of the scalar loop. The class (b) diagrams have all the gluon lines on the outside of the scalar loop.

To illustrate the utility of scalar-loop contributions in constructing the gluon-loop one, consider the two-loop four-gluon amplitudes with a single scalar loop. Figure 4 shows that there are two different classes of planar diagrams, divided according to whether or not an internal gluon line runs inside the scalar loop. For the case of scalars in the fundamental representation of $S U\left(N_{c}\right)$, the contributions proportional to the color trace $\operatorname{Tr}\left[T^{a_{1}} T^{a_{2}} T^{a_{3}} T^{a_{4}}\right]$ are

$$
\operatorname{Tr}\left[T^{a_{1}} T^{a_{2}} T^{a_{3}} T^{a_{4}}\right]\left[N_{c} A_{4}^{2-\text { loop, (b) }}-\frac{1}{N_{c}} A_{4}^{2 \text {-loop,(a) }}\right],
$$

where $N_{c}$ is the number of colors, and the labels (a) and (b) correspond to the diagram classes depicted in fig. 4. The amplitudes are color-stripped; that is, they should be computed using colorordered Feynman rules [1] from which color factors have been removed. Because the diagrams of class (a) and (b) enter eq. (3.1) with different powers of $N_{c}$, they are separately gauge invariant and may be computed independently. For scalars in the adjoint representation the two contributions are still separately gauge invariant, but there is no relative factor of $-N_{c}^{2}$. The leading-color contribution proportional to the same color trace is then

$$
\operatorname{Tr}\left[T^{a_{1}} T^{a_{2}} T^{a_{3}} T^{a_{4}}\right] N_{c}^{2}\left[A_{4}^{2-\text { loop,(b) }}+A_{4}^{2-\text { loop, (a) }}\right] .
$$

We begin our evaluation of scalar-loop contributions with the $s$-channel double two-particle cut for the subleading-color term in fig. 4(a). This is given by the product (2.3) where only scalars cross the generalized cuts,

$$
\begin{aligned}
A_{4}^{2 \text {-loop },(\mathrm{a})} & \left.\left(1^{+}, 2^{+}, 3^{+}, 4^{+}\right)\right|_{2 \times 2 \text {-cut }} \\
& =A_{4}^{\text {tree }}\left(1^{+}, 2^{+},-\ell_{2}^{s},-\ell_{1}^{s}\right) \times A_{4}^{\text {tree }}\left(\ell_{1}^{s}, \ell_{2}^{s},-\ell_{3}^{s},-\ell_{4}^{s}\right) \times A_{4}^{\text {tree }}\left(\ell_{4}^{s}, \ell_{3}^{s}, 3^{+}, 4^{+}\right) \\
& =i \frac{[12][34]}{\langle 12\rangle\langle 34\rangle}\left(\frac{s_{12} \lambda_{1}^{2} \lambda_{4}^{2}}{\left(\ell_{1}-k_{1}\right)^{2}\left(\ell_{1}-\ell_{4}\right)^{2}\left(\ell_{4}+k_{4}\right)^{2}}+\frac{1}{2} \frac{\lambda_{1}^{2} \lambda_{4}^{2}}{\left(\ell_{1}-k_{1}\right)^{2}\left(\ell_{4}+k_{4}\right)^{2}}\right),
\end{aligned}
$$

where $s_{i j}=\left(k_{i}+k_{j}\right)^{2}$. The vectors $\vec{\lambda}_{i}$ represent the $(-2 \epsilon)$-dimensional components of the loop momenta $\ell_{i}$; that is, $\ell_{i} \equiv \ell_{i}^{[4]}+\lambda_{i}$, where $\ell_{i}^{[4]}$ has only four-dimensional components. Superscripts \pm 
label helicities of external gluons, while a superscript $s$ indicates that a cut leg is a scalar. (A cut momentum without a superscript will implicitly denote a gluon.) We used the color-ordered [1] tree amplitudes (or partial amplitudes),

$$
\begin{aligned}
& A_{4}^{\text {tree }}\left(1^{+}, 2^{+},-\ell_{2}^{s},-\ell_{1}^{s}\right)=i \lambda_{1}^{2} \frac{[12]}{\langle 12\rangle} \frac{1}{\left(\ell_{1}-k_{1}\right)^{2}}, \\
& A_{4}^{\text {tree }}\left(\ell_{1}^{s}, \ell_{2}^{s},-\ell_{3}^{s},-\ell_{4}^{s}\right)=-i\left(\frac{s_{12}}{\left(\ell_{1}-\ell_{4}\right)^{2}}+\frac{1}{2}\right)
\end{aligned}
$$

$A_{4}^{\text {tree }}\left(\ell_{4}^{s}, \ell_{3}^{s}, 3^{+}, 4^{+}\right)$can be obtained by relabeling $A_{4}^{\text {tree }}\left(1^{+}, 2^{+},-\ell_{2}^{s},-\ell_{1}^{s}\right)$. We have expressed the amplitudes in terms of spinor inner products [1] which are denoted by $\langle i j\rangle=\left\langle i^{-} \mid j^{+}\right\rangle$and $[i j]=$ $\left\langle i^{+} \mid j^{-}\right\rangle$, where $\left|i^{ \pm}\right\rangle$are four-dimensional massless Weyl spinors of momentum $k_{i}$, labeled with the sign of the helicity.

As a working hypothesis, from eq. (3.3) we take the subleading-color planar amplitude to be ${ }^{1}$

$$
\begin{gathered}
A_{4}^{2 \text {-loop },(\mathrm{a})}\left(1^{+}, 2^{+}, 3^{+}, 4^{+}\right)=i \frac{[12][34]}{\langle 12\rangle\langle 34\rangle}\left[s_{12} \mathcal{I}_{4}^{\mathrm{P}}\left[\lambda_{p}^{2} \lambda_{q}^{2}\right]\left(s_{12}, s_{23}\right)\right. \\
+\frac{1}{2} \mathcal{I}_{4}^{\text {bow-tie }}\left[\lambda_{p}^{2} \lambda_{q}^{2}\right]\left(s_{12}\right) \\
\left.+\left(s_{12} \leftrightarrow s_{23}\right)\right]
\end{gathered}
$$

where the two terms correspond to the two terms in the second equation of eq. (3.3),

$$
\begin{aligned}
\mathcal{I}_{4}^{\mathrm{P}}[\mathcal{P} & \left.\left(\lambda_{i}, p, q, k_{i}\right)\right]\left(s_{12}, s_{23}\right) \\
& \equiv \int \frac{d^{D} p}{(2 \pi)^{D}} \frac{d^{D} q}{(2 \pi)^{D}} \frac{\mathcal{P}\left(\lambda_{i}, p, q, k_{i}\right)}{p^{2} q^{2}(p+q)^{2}\left(p-k_{1}\right)^{2}\left(p-k_{1}-k_{2}\right)^{2}\left(q-k_{4}\right)^{2}\left(q-k_{3}-k_{4}\right)^{2}}
\end{aligned}
$$

is the planar double box integral displayed in fig. $5(\mathrm{a})$, and the vectors $\vec{\lambda}_{p}, \vec{\lambda}_{q}$ represent the $(-2 \epsilon)$ dimensional components of the loop momenta $p$ and $q$. The numerator factor $\mathcal{P}\left(\lambda_{i}, p, q, k_{i}\right)$ is a polynomial in the momenta. The 'bow-tie' integral $\mathcal{I}_{4}^{\text {bow-tie }}$ shown in fig. 6 is defined by

$$
\begin{aligned}
\mathcal{I}_{4}^{\text {bow-tie }}[\mathcal{P} & \left.\left(\lambda_{i}, p, q, k_{i}\right)\right]\left(s_{12}\right) \\
& \equiv \int \frac{d^{D} p}{(2 \pi)^{D}} \frac{d^{D} q}{(2 \pi)^{D}} \frac{\mathcal{P}\left(\lambda_{i}, p, q, k_{i}\right)}{p^{2} q^{2}\left(p-k_{1}\right)^{2}\left(p-k_{1}-k_{2}\right)^{2}\left(q-k_{4}\right)^{2}\left(q-k_{3}-k_{4}\right)^{2}} .
\end{aligned}
$$

The one 'missing' propagator, compared with $\mathcal{I}_{4}^{\text {P }}$, makes $\mathcal{I}_{4}^{\text {bow-tie }}$ much simpler to evaluate, since it is a product of two independent one-loop integrals. Although it is not manifest, the overall spinor prefactor is symmetric under all 4 ! permutations of the external legs, including the cyclic relabeling, $k_{1} \rightarrow k_{2} \rightarrow k_{3} \rightarrow k_{4} \rightarrow k_{1}$. Hence the amplitude (3.5), including the term with $s_{12}$ and $s_{23}$ exchanged, is cyclicly invariant too. The exchange term generates the proper double two-particle cut in the $t$-channel.

Cutting a line forces the corresponding propagator to be present, uncanceled, in all integral functions. In general, an ansatz for an amplitude based on a limited set of cuts will fail to capture integral functions which are missing some or all of the cut propagators. As one refines the ansatz by investigating additional cuts, it may be necessary to add in integral functions that do not have cuts

\footnotetext{
${ }^{1}$ These bare amplitudes should be multiplied by the scale factor $\left(\mu_{0}^{2}\right)^{2 \epsilon}$, and after renormalization instead by the scale $\left(\mu_{R}^{2}\right)^{2 \epsilon}$. Here we take these scales to equal unity except where noted.
} 


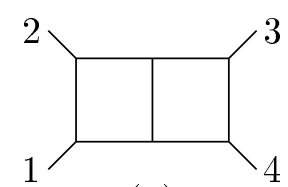

(a)

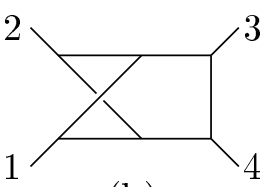

(b)

Figure 5: The planar and non-planar double box integrals.

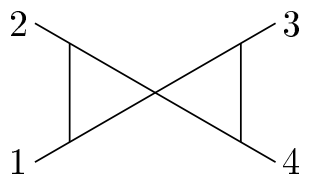

Figure 6: The 'bow-tie' integral appearing in eq. (3.5).

in previously-investigated channels. For example, the integral depicted in fig. 7 contains only threeparticle cuts and would not be detected in two-particle cuts. However, for the planar amplitude under consideration, it turns out that such integrals do not appear, and the double two-particle cuts are in fact sensitive to all functions appearing in the amplitude.

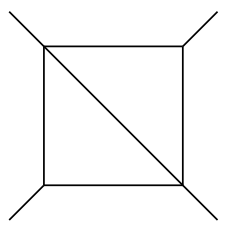

Figure 7: An integral function containing only three particle cuts.

To verify that eq. (3.5) is indeed the complete amplitude corresponding to the class (a) diagrams in fig. 8 we must evaluate the two- and three-particle cuts. To evaluate the two-particle cuts we need the first of the tree amplitudes given in eq. (3.4), as well as the subleading-in-color one-loop amplitude (see fig. 8(a) for a representative diagram),

$$
A_{4}^{1 \text {-loop, (a) }}\left(1^{+}, 2^{+}, 3^{s}, 4^{s}\right)=-\frac{[12]}{\langle 12\rangle}\left[s_{12} \mathcal{I}_{4}^{1-\text { loop }}\left[\lambda_{p}^{2}\right]\left(s_{12}, s_{23}\right)+\frac{1}{2} \mathcal{I}_{3}^{1-\text { loop },(4)}\left[\lambda_{p}^{2}\right]\left(s_{12}\right)\right]
$$

(We compute all required one-loop amplitudes from their $s$ - and $t$-channel cuts.) We have taken the momenta of the external legs 1 and 2 to be four-dimensional while the momenta of legs 3 and 4 are $D$-dimensional, so that they can be inserted into the two-particle cuts. The one-loop box integral in eq. (3.8) is defined by

$$
\mathcal{I}_{4}^{1-\text { loop }}\left[\mathcal{P}\left(\lambda_{p}, p, k_{i}\right)\right] \equiv \int \frac{d^{D} p}{(2 \pi)^{D}} \frac{\mathcal{P}\left(\lambda_{p}, p, k_{i}\right)}{p^{2}\left(p-k_{1}\right)^{2}\left(p-k_{1}-k_{2}\right)^{2}\left(p+k_{4}\right)^{2}}
$$



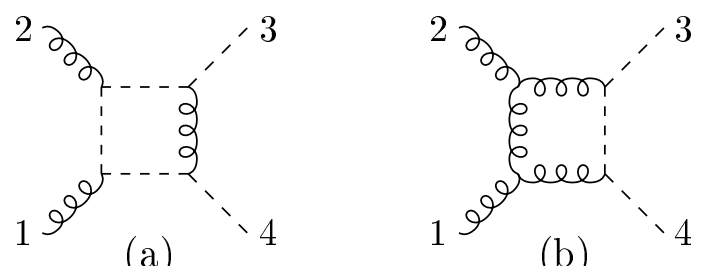

Figure 8: Representative planar diagrams for the subleading-in-color (a) and leading-in-color (b) one-loop amplitudes for two gluons and two scalars. The diagrams are divided into the two classes according to whether or not there is an internal gluon line to the right of the scalar line.

The triangle integral $\mathcal{I}_{3}^{1 \text {-loop,(4) }}$ is obtained by removing the $1 /\left(p+k_{4}\right)^{2}$ propagator from eq. (3.9). Taking the two-particle cut of the ansatz (3.5) we obtain

$$
\begin{aligned}
\left.A_{4}^{2 \text {-loop },(a)}\left(1^{+}, 2^{+}, 3^{+}, 4^{+}\right)\right|_{2 \text {-cut }} & =A_{4}^{\text {tree }}\left(1^{+}, 2^{+},-\ell_{2}^{s},-\ell_{1}^{s}\right) \times A_{4}^{1 \text {-loop },(\mathrm{a})}\left(\ell_{2}^{s}, \ell_{1}^{s}, 3^{+}, 4^{+}\right) \\
& +A_{4}^{1 \text {-loop },(\mathrm{a})}\left(1^{+}, 2^{+},-\ell_{2}^{s},-\ell_{1}^{s}\right) \times A_{4}^{\text {tree }}\left(\ell_{2}^{s}, \ell_{1}^{s}, 3^{+}, 4^{+}\right),
\end{aligned}
$$

as required. The two-particle cuts work simply in this example because all terms in the one-loop amplitude (3.8) could be detected via the $s$-channel cut.

The three-particle cuts are more intricate, but may be handled numerically. For the $s$-channel case, we need the product of tree amplitudes,

$$
A_{5}^{\text {tree }}\left(1^{+}, 2^{+},-\ell_{3}^{s},-\ell_{2}^{\mu},-\ell_{1}^{s}\right) \times P_{\mu \nu}\left(\ell_{2}, r\right) \times A_{5}^{\text {tree }}\left(\ell_{1}^{s}, \ell_{2}^{\nu}, \ell_{3}^{s}, 3^{+}, 4^{+}\right),
$$

where $\mu, \nu$ are Lorentz indices for the single gluon line crossing the cut, and the physical state projector $P_{\mu \nu}$ is defined in eq. (2.5). If our ansatz (3.5) is correct, then the cut expression (3.11) should be equal to

$$
\begin{aligned}
& i \frac{[12][34]}{\langle 12\rangle\langle 34\rangle} \lambda_{1}^{2} \lambda_{3}^{2}\left[\frac{s_{23}}{\left(\ell_{1}-k_{1}\right)^{2}\left(\ell_{3}-k_{2}\right)^{2}\left(\ell_{3}+k_{3}\right)^{2}\left(\ell_{1}+k_{4}\right)^{2}}\right. \\
& +\frac{s_{12}}{\left(\ell_{1}+\ell_{2}\right)^{2}\left(\ell_{3}-k_{2}\right)^{2}\left(\ell_{2}+\ell_{3}\right)^{2}\left(\ell_{1}+k_{4}\right)^{2}} \\
& \left.+\frac{s_{12}}{\left(\ell_{1}+\ell_{2}\right)^{2}\left(\ell_{3}+k_{3}\right)^{2}\left(\ell_{2}+\ell_{3}\right)^{2}\left(\ell_{1}-k_{1}\right)^{2}}\right] \text {, }
\end{aligned}
$$

which is obtained by cutting the two planar double box integrals in eq. (3.5) as shown in fig. 9. The bow-tie integrals do not contribute to three-particle cuts. The equality of these expressions should hold before any loop or phase-space integration is performed.

The expressions for the cuts are smooth analytic functions with no explicit dependence on the dimension $D$ of the loop-momentum vectors. It is therefore sufficient to verify the cuts for integer values of $D$, with $D>4$, where numerical evaluation is straightforward. We randomly generate $D$-vectors satisfying the appropriate cut kinematics - in this case, seven null-vectors $\left\{k_{1}, k_{2}, k_{3}, k_{4}, \ell_{1}, \ell_{2}, \ell_{3}\right\}$ obeying

$$
k_{1}+k_{2}-\ell_{1}-\ell_{2}-\ell_{3}=k_{3}+k_{4}+\ell_{1}+\ell_{2}+\ell_{3}=0
$$

where the $k_{i}$ are four-dimensional, i.e., they have vanishing extra-dimensional components. We then check whether the cuts agree with our ansatz at this point in phase space, in this case by comparing 

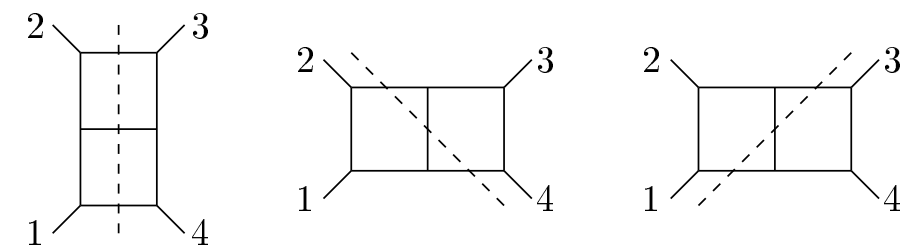

Figure 9: The s-channel three-particle cuts of the class (a) scalar contribution in eq. (3.5), and of the leading-color pure gluon contribution in eq. (4.1). The dashed lines represent the cuts.

numerical values for eqs. (3.11) and (3.12). Because no integration is required, the check can be performed to very high accuracy (25 or more digits), and repeated quickly for other phase-space points. As mentioned previously, we take the dimension $D_{s}$, which appears in the number of gluon states circulating in the loop, to be independent from the dimension $D$ of the loop momentum vectors. To distinguish between e.g. $\left(\lambda_{1} \cdot \lambda_{2}\right)^{2}$ and $\lambda_{1}^{2} \lambda_{2}^{2}$ (the most subtle degeneracy arising at two loops) it is necessary to choose $D \geq 6$.

Together with the two-particle cuts, the numerical verification of the three-particle cuts proves that the ansatz (3.5) does indeed give the complete subleading-color contribution to the color coefficient $\operatorname{Tr}\left[T^{a_{1}} T^{a_{2}} T^{a_{3}} T^{a_{4}}\right]$ for the case of a scalar in the loop.

Following a similar procedure we have also obtained the results for the leading-color contributions, obtained from the diagrams of the type displayed in fig. 4(b). The color-stripped amplitude for case (b) is,

$$
\begin{aligned}
A_{4}^{2-\text { loop },(\mathrm{b})}\left(1^{+}, 2^{+}, 3^{+}, 4^{+}\right)= & i \frac{[12][34]}{\langle 12\rangle\langle 34\rangle}\left[s_{12} \mathcal{I}_{4}^{\mathrm{P}}\left[\lambda_{p}^{2} \lambda_{p+q}^{2}\right]\left(s_{12}, s_{23}\right)\right. \\
& +\frac{\left(D_{s}-2\right)}{s_{12}} \mathcal{I}_{4}^{\text {bow-tie }}\left[\lambda_{p}^{2} \lambda_{q}^{2}\left((p+q)^{2}+s_{12}\right)\right]\left(s_{12}, s_{23}\right) \\
& \left.+4 \mathcal{I}_{4}^{\text {bow-tie }}\left[\lambda_{p}^{2}\left(\lambda_{p} \cdot \lambda_{q}\right)\right]\left(s_{12}\right)+\text { cyclic perms of }(1,2,3,4)\right] .
\end{aligned}
$$

In this case the number of gluonic states $\left(D_{s}-2\right)$ circulating in the loop appears. Although some of the terms in eq. (3.14) vanish under integration, we have kept them because they do not vanish in the cuts before integration and are therefore important for verifying the cuts numerically.

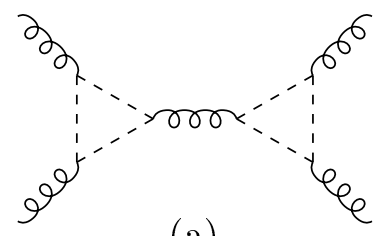

(a)

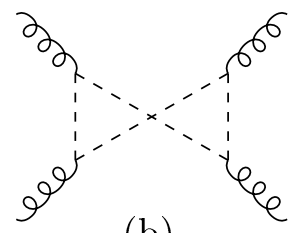

(b)

Figure 10: Representative planar diagrams with two scalar loops. The ones in (a) have two distinct loops connected by a gluon line; the ones in (b) have a quartic scalar interaction.

The two-loop four-gluon amplitude with scalars present can also receive contributions with two 
scalar loops. Figure 10(a) shows a class that is present just due to the gauge interaction. Because supersymmetric gauge theories with scalars always include quartic scalar interactions (arising from $D$-terms), it is useful to also calculate the class shown in fig. 10(b). Both types of contributions are simple,

$$
\begin{aligned}
& A_{4}^{\text {double-scalar,(a) }}\left(1^{+}, 2^{+}, 3^{+}, 4^{+}\right) \propto i \frac{[12][34]}{\langle 12\rangle\langle 34\rangle} \frac{1}{s_{12}} \mathcal{I}_{4}^{\text {bow-tie }}\left[\lambda_{p}^{2} \lambda_{q}^{2}\left((p+q)^{2}+\frac{1}{2} s_{12}\right)\right]\left(s_{12}, s_{23}\right), \\
& A_{4}^{\text {double-scalar,(b) }}\left(1^{+}, 2^{+}, 3^{+}, 4^{+}\right) \propto i \frac{[12][34]}{\langle 12\rangle\langle 34\rangle} \mathcal{I}_{4}^{\text {bow-tie }}\left[\lambda_{p}^{2} \lambda_{q}^{2}\right]\left(s_{12}\right) .
\end{aligned}
$$

The precise normalization is unimportant; in constructing the pure gluon ansatz we shall add these terms to eqs. (3.5) and (3.14) with coefficients to be determined later.

\section{The pure gluon amplitude}

We now turn to the two-loop pure gluon amplitude $\mathcal{A}_{4}^{2-\text { loop }}\left(1^{+}, 2^{+}, 3^{+}, 4^{+}\right)$, which is the main subject of this paper. It is convenient to begin with the leading-color contribution generated by planar graphs.

\subsection{Leading color}

The planar amplitudes with internal scalars from section 3 serve as a guide toward constructing the planar pure gluon contributions. We write an ansatz in terms of loop integrals by taking a linear combination of the above results for one scalar in the loop, plus contributions with two independent scalar loops. This turns out not to be the complete answer so an additional polynomial in the $\lambda_{i}$ is required, whose form is not too hard to guess. We numerically evaluate the $D$-dimensional cuts at a number of different phase-space points, and use this information to solve for the unknown (but kinematically constant) coefficients of possible additional terms. In this way we obtain a simple representation of the planar pure gluon amplitude in terms of loop integrals. The form of the planar amplitude then suggests an ansatz for remaining non-planar contributions, which we again verify numerically from the cuts.

In somewhat more detail, our starting point is the contribution of a single adjoint scalar loop, eq. (3.2). We multiply this contribution by the number of gluon states, $D_{s}-2$. This would produce the complete answer at one loop, but here we must make two corrections:

- a factor of $1 / 2$ for terms proportional to $\left(D_{s}-2\right)^{2}$, from different combinatorics of contributions in which the two loops have no common propagator, as in fig. 10(a).

- the addition of a term (with an unknown coefficient) induced by a quartic scalar interaction, as in fig. 10(b).

Even with these corrections, the ansatz does not quite work. However, it does satisfy the double two-particle and three-particle cuts in $D=4$ (i.e., when all $(-2 \epsilon)$-dimensional components are set to zero) and also in $D=5$. This provides a strong clue to the form of the additional term; it must 
be proportional to powers of $\vec{\lambda}_{i}$, and it must vanish when all the vectors $\vec{\lambda}_{i}$ are parallel. The missing term, determined numerically, is the double box planar integral with numerator $16\left(D_{s}-2\right) s_{12}\left(\left(\lambda_{p}\right.\right.$. $\left.\left.\lambda_{q}\right)^{2}-\lambda_{p}^{2} \lambda_{q}^{2}\right)$. We then find for the pure gluon leading-color all-plus helicity amplitude in eq. (2.4),

$$
A_{4 ; 1 ; 1}^{\mathrm{LC}}\left(1^{+}, 2^{+}, 3^{+}, 4^{+}\right)=A_{1234}^{\mathrm{P}}+A_{2341}^{\mathrm{P}},
$$

where the 'primitive' planar amplitude $A_{1234}^{\mathrm{P}}$ is analogous to the one appearing in the $N=4$ superYang-Mills case [7],

$$
\begin{aligned}
A_{1234}^{\mathrm{P}}=i \frac{[12][34]}{\langle 12\rangle\langle 34\rangle}\left\{s_{12}\right. & \mathcal{I}_{4}^{\mathrm{P}}\left[\left(D_{s}-2\right)\left(\lambda_{p}^{2} \lambda_{q}^{2}+\lambda_{p}^{2} \lambda_{p+q}^{2}+\lambda_{q}^{2} \lambda_{p+q}^{2}\right)+16\left(\left(\lambda_{p} \cdot \lambda_{q}\right)^{2}-\lambda_{p}^{2} \lambda_{q}^{2}\right)\right]\left(s_{12}, s_{23}\right) \\
& +4\left(D_{s}-2\right) \mathcal{I}_{4}^{\text {bow-tie }}\left[\left(\lambda_{p}^{2}+\lambda_{q}^{2}\right)\left(\lambda_{p} \cdot \lambda_{q}\right)\right]\left(s_{12}\right) \\
& \left.+\frac{\left(D_{s}-2\right)^{2}}{s_{12}} \mathcal{I}_{4}^{\text {bow-tie }}\left[\lambda_{p}^{2} \lambda_{q}^{2}\left((p+q)^{2}+s_{12}\right)\right]\left(s_{12}, s_{23}\right)\right\} .
\end{aligned}
$$

\subsection{Subleading color}

We may observe that all terms in $A^{\mathrm{LC}}$ have two-particle cuts in exactly one channel. If we assume that this property also holds for the subleading-color contribution, then performing the sewings implied by eq. (2.1), we find that full two-loop amplitude can be written in the following form

$$
\mathcal{A}_{4}^{2-\text { loop }}\left(1^{+}, 2^{+}, 3^{+}, 4^{+}\right)=g^{6}\left[C_{1234}^{\mathrm{P}} A_{1234}^{\mathrm{P}}+C_{3421}^{\mathrm{P}} A_{3421}^{\mathrm{P}}+C_{12 ; 34}^{\mathrm{NP}} A_{12 ; 34}^{\mathrm{NP}}+C_{34 ; 21}^{\mathrm{NP}} A_{34 ; 21}^{\mathrm{NP}}+\mathcal{C}(234)\right],
$$

where ' $+\mathcal{C}(234)$ ' instructs one to add the two non-trivial cyclic permutations of $(2,3,4)$. The values of the color coefficients may be read off directly from fig. 5. For example, $C_{1234}^{\mathrm{P}}$ is the color factor obtained from diagram (a) by dressing each vertex with an $\tilde{f}^{a b c}$, where

$$
\tilde{f}^{a b c} \equiv i \sqrt{2} f^{a b c}=\operatorname{Tr}\left(\left[T^{a}, T^{b}\right] T^{c}\right),
$$

and dressing each internal line with a $\delta^{a b}$. Similarly, $C_{12 ; 34}^{\mathrm{NP}}$ is obtained by dressing diagram (b). The symmetries of the color factors can also be read off the diagrams:

$$
\begin{gathered}
C_{1234}^{\mathrm{P}}=C_{3412}^{\mathrm{P}}=C_{2143}^{\mathrm{P}}=C_{4321}^{\mathrm{P}}, \\
C_{12 ; 34}^{\mathrm{NP}}=C_{21 ; 34}^{\mathrm{NP}}=C_{12 ; 43}^{\mathrm{NP}}=C_{21 ; 43}^{\mathrm{NP}} .
\end{gathered}
$$

The corresponding planar and non-planar primitive amplitudes share the same symmetries with their associated color factors. Due to these symmetries, $\mathcal{A}_{4}^{2-\text { loop }}\left(1^{+}, 2^{+}, 3^{+}, 4^{+}\right)$has the required total $\left(S_{4}\right)$ permutation symmetry, even though only six permutations appear explicitly in eq. (4.3).

In the amplitude (4.3) no ultraviolet subtraction has been performed. The subtraction in the $\overline{\mathrm{MS}}$ scheme is given below in eq. (5.21).

The one-channel assumption about the two-particle cuts is non-trivial and is not expected to hold for general helicity amplitudes. This property and the decomposition of eq. (4.3) are also satisfied by the $N=4$ supersymmetric amplitude [7,9]. One may verify the validity of the assumption $a$ posteriori by checking the two- and three-particle cuts, eqs. (2.1) and (2.2). Although it might appear 
that the expression (4.3) does not assign the proper color factors to bow-tie integral contributions, the unwanted terms (the subleading-color parts of $C^{\mathrm{P}}$ ) cancel in the permutation sum. The partial amplitudes appearing in the standard color decomposition (2.4) can be expressed in terms of $A^{\mathrm{P}}$ and $A^{\mathrm{NP}}$. The equations are identical to those for the $N=4$ supersymmetric case discussed in ref. [7].

From the point of view of the two-particle cuts, the planar amplitude arises from sewing a fourpoint color-ordered tree to a color-ordered one-loop amplitude, where the sewn legs are adjacent in both amplitudes. In other terms in eq. (2.1), the permutation of legs on the one-loop amplitude $\mathcal{A}_{4}^{1-\text { loop }}$ results in sewing non-adjacent (diagonally opposite) legs to the tree. This gives rise to a primitive amplitude built out of non-planar two-loop integrals,

$A_{12 ; 34}^{\mathrm{NP}}=i \frac{[12][34]}{\langle 12\rangle\langle 34\rangle} s_{12} \mathcal{I}_{4}^{\mathrm{NP}}\left[\left(D_{s}-2\right)\left(\lambda_{p}^{2} \lambda_{q}^{2}+\lambda_{p}^{2} \lambda_{p+q}^{2}+\lambda_{q}^{2} \lambda_{p+q}^{2}\right)+16\left(\left(\lambda_{p} \cdot \lambda_{q}\right)^{2}-\lambda_{p}^{2} \lambda_{q}^{2}\right)\right]\left(s_{12}, s_{23}\right)$,

where the non-planar double box integral, depicted in fig. 5(b), is given by

$$
\begin{aligned}
& \mathcal{I}_{4}^{\mathrm{NP}}\left[\mathcal{P}\left(\lambda_{i}, p, q, k_{i}\right)\right]\left(s_{12}, s_{23}\right) \\
& \quad \equiv \int \frac{d^{D} p}{(2 \pi)^{D}} \frac{d^{D} q}{(2 \pi)^{D}} \frac{\mathcal{P}\left(\lambda_{i}, p, q, k_{i}\right)}{p^{2} q^{2}(p+q)^{2}\left(p-k_{1}\right)^{2}\left(q-k_{2}\right)^{2}\left(p+q+k_{3}\right)^{2}\left(p+q+k_{3}+k_{4}\right)^{2}} .
\end{aligned}
$$

Note that $A_{12 ; 34}^{\mathrm{NP}}$ is symmetric under $k_{1} \leftrightarrow k_{2}$, and independently under $k_{3} \leftrightarrow k_{4}$. The non-planar amplitude has no bow-tie contributions; however, the term proportional to $\left(\left(\lambda_{p} \cdot \lambda_{q}\right)^{2}-\lambda_{p}^{2} \lambda_{q}^{2}\right)$ persists.

To verify that the expressions in eqs. (4.3), (4.2) and (4.6) give the correct full gluon amplitude, we have evaluated all the independent cuts. Consider, for example, the leading-color planar contribution proportional to the color factor $N_{c}^{2} \operatorname{Tr}\left[T^{a_{1}} T^{a_{2}} T^{a_{3}} T^{a_{4}}\right]$, which is given by eq. (4.1). For this contribution, the $s_{12}$-channel three-particle cut integrand should be equal to the product of color-ordered five-gluon tree-level partial amplitudes,

$$
A_{5}^{\text {tree }}\left(1^{+}, 2^{+},-\ell_{3}^{\rho},-\ell_{2}^{\nu},-\ell_{1}^{\mu}\right) \times P_{\mu \alpha}\left(\ell_{1}, r\right) P_{\nu \beta}\left(\ell_{2}, r\right) P_{\rho \gamma}\left(\ell_{3}, r\right) \times A_{5}^{\text {tree }}\left(\ell_{1}^{\alpha}, \ell_{2}^{\beta}, \ell_{3}^{\gamma}, 3^{+}, 4^{+}\right) .
$$

We have verified numerically that the product of tree amplitudes (4.8) is equal to the three-particle cut of the leading-color expression (4.1), given by

$$
\begin{aligned}
& i \frac{[12][34]}{\langle 12\rangle\langle 34\rangle}\left[\left(D_{s}-2\right)\left(\lambda_{1}^{2} \lambda_{2}^{2}+\lambda_{1}^{2} \lambda_{3}^{2}+\lambda_{2}^{2} \lambda_{3}^{2}\right)+16\left(\left(\lambda_{1} \cdot \lambda_{3}\right)^{2}-\lambda_{1}^{2} \lambda_{3}^{2}\right)\right] \\
& \quad \times\left[\frac{s_{23}}{\left(\ell_{1}-k_{1}\right)^{2}\left(\ell_{3}-k_{2}\right)^{2}\left(\ell_{3}+k_{3}\right)^{2}\left(\ell_{1}+k_{4}\right)^{2}}+\frac{s_{12}}{\left(\ell_{1}+\ell_{2}\right)^{2}\left(\ell_{3}-k_{2}\right)^{2}\left(\ell_{2}+\ell_{3}\right)^{2}\left(\ell_{1}+k_{4}\right)^{2}}\right. \\
& \left.+\frac{s_{12}}{\left(\ell_{1}+\ell_{2}\right)^{2}\left(\ell_{3}+k_{3}\right)^{2}\left(\ell_{2}+\ell_{3}\right)^{2}\left(\ell_{1}-k_{1}\right)^{2}}\right] .
\end{aligned}
$$

The three different terms in the last factor in eq. (4.9) originate from the two cut planar double box integrals in the same way as in the scalar example (3.12), as illustrated in fig. 9.

As a second example of a $s_{12}$-channel three-particle cut, but one that is sensitive to non-planar (subleading-color) contributions, we evaluate the product

$$
A_{5}^{\text {tree }}\left(1^{+},-\ell_{3}^{\rho},-\ell_{2}^{\nu}, 2^{+},-\ell_{1}^{\mu}\right) \times P_{\mu \alpha}\left(\ell_{1}, r\right) P_{\nu \beta}\left(\ell_{2}, r\right) P_{\rho \gamma}\left(\ell_{3}, r\right) \times A_{5}^{\text {tree }}\left(\ell_{1}^{\alpha}, \ell_{2}^{\beta}, \ell_{3}^{\gamma}, 3^{+}, 4^{+}\right)
$$


numerically and find that it equals

$$
\begin{aligned}
& i \frac{[12][34]}{\langle 12\rangle\langle 34\rangle}\left[\left(D_{s}-2\right)\left(\lambda_{1}^{2} \lambda_{2}^{2}+\lambda_{1}^{2} \lambda_{3}^{2}+\lambda_{2}^{2} \lambda_{3}^{2}\right)+16\left(\left(\lambda_{1} \cdot \lambda_{3}\right)^{2}-\lambda_{1}^{2} \lambda_{3}^{2}\right)\right] \\
& \quad \times\left[-\frac{s_{13}}{\left(\ell_{3}-k_{1}\right)^{2}\left(\ell_{1}-k_{2}\right)^{2}\left(\ell_{3}+k_{3}\right)^{2}\left(\ell_{1}+k_{4}\right)^{2}}-\frac{s_{13}}{\left(\ell_{3}-k_{1}\right)^{2}\left(\ell_{2}-k_{2}\right)^{2}\left(\ell_{3}+k_{3}\right)^{2}\left(\ell_{1}+k_{4}\right)^{2}}\right. \\
& \quad+\frac{s_{23}}{\left(\ell_{1}-k_{1}\right)^{2}\left(\ell_{2}-k_{2}\right)^{2}\left(\ell_{3}+k_{3}\right)^{2}\left(\ell_{1}+k_{4}\right)^{2}}+\frac{s_{12}}{\left(\ell_{3}-k_{1}\right)^{2}\left(\ell_{2}-k_{2}\right)^{2}\left(\ell_{2}+\ell_{3}\right)^{2}\left(\ell_{1}+k_{4}\right)^{2}} \\
& \quad-\frac{s_{12}}{\left(\ell_{1}-k_{1}\right)^{2}\left(\ell_{2}-k_{2}\right)^{2}\left(\ell_{3}+k_{3}\right)^{2}\left(\ell_{1}+\ell_{2}\right)^{2}}-\frac{s_{12}}{\left(\ell_{1}-k_{1}\right)^{2}\left(\ell_{2}+\ell_{3}\right)^{2}\left(\ell_{3}+k_{3}\right)^{2}\left(\ell_{1}+\ell_{2}\right)^{2}} \\
& \left.\quad-\frac{s_{3}}{\left(\ell_{1}-k_{2}\right)^{2}\left(\ell_{2}+\ell_{3}\right)^{2}\left(\ell_{3}+k_{3}\right)^{2}\left(\ell_{1}+\ell_{2}\right)^{2}}\right] .
\end{aligned}
$$

The latter candidate expression is found by extracting from eq. (4.3) the seven terms with the correct color ordering to contribute to the cut (4.10). (Three different planar double box integrals and three different non-planar ones appear; one of the non-planar integrals can be cut two separate ways. Minus signs are due to the antisymmetry of the structure constants in the color factors.) More generally, we have verified that the amplitude (4.3) has the correct $D$-dimensional two- and three-particle cuts in all channels, proving it to be correct.

We have also shown that the double two-particle and three-particle cuts of the Feynman gauge Feynman diagrams, including the ghosts, match the cuts of our expressions. In performing this consistency check there is no need to include physical state projectors on the intermediate gluon lines; the ghosts automatically cancel the unwanted longitudinal modes. We carried out the comparison numerically for specific values of the (cut) loop momenta. This provides a non-trivial check that our expressions are in one-to-one correspondence with the results that one would obtain via a direct evaluation of the Feynman diagrams.

In the appendix we perform the final step in a closed-form evaluation of the two-loop amplitude: We present the values of the integrals appearing in the amplitudes, expanded in $\epsilon$ through $\mathcal{O}\left(\epsilon^{0}\right)$ and expressed in terms of polylogarithms. The bow-tie integrals are rather simple to evaluate since they are just products of one-loop integrals. The planar and non-planar double box integrals are much more difficult to obtain, because they have poles up to order $1 / \epsilon^{2}$ and an intricate analytic structure (especially the non-planar cases).

\section{$5 \quad$ Divergences}

In this section we compare the divergence structure of the all-plus helicity amplitude (4.3) against the one expected from general principles. Catani has previously presented a universal factorization formula for the infrared divergent parts of dimensionally regulated, renormalized two-loop amplitudes [12]. In the all-plus case, the divergence structure is relatively simple, due to the vanishing of the corresponding tree-level helicity amplitude; both the ultraviolet and infrared divergences are essentially the same ones encountered at one loop [13]. Nevertheless, we find it convenient to use Catani's formalism, because of its more general applicability.

In the color space operator language used by Catani, the infrared divergences of a renormalized 
two-loop amplitude are,

$$
\begin{aligned}
\left|\mathcal{M}_{n}^{(2)}\left(\mu_{R}^{2} ;\{p\}\right)\right\rangle_{\mathrm{RS}}=\boldsymbol{I}^{(1)} & \left(\epsilon, \mu_{R}^{2} ;\{p\}\right)\left|\mathcal{M}_{n}^{(1)}\left(\mu_{R}^{2} ;\{p\}\right)\right\rangle_{\mathrm{RS}} \\
& +\boldsymbol{I}_{\mathrm{RS}}^{(2)}\left(\epsilon, \mu_{R}^{2} ;\{p\}\right)\left|\mathcal{M}_{n}^{(0)}\left(\mu_{R}^{2} ;\{p\}\right)\right\rangle_{\mathrm{RS}}+\text { finite }
\end{aligned}
$$

where $\left|\mathcal{M}_{n}^{(L)}\left(\mu_{R}^{2} ;\{p\}\right)\right\rangle_{\mathrm{RS}}$ is a color space vector representing the renormalized $L$ loop amplitude. The subscript RS stands for the choice of renormalization scheme, and $\mu_{R}$ is the renormalization scale (which we have set to unity elsewhere in the paper). These color space vectors give the amplitudes via,

$$
\mathcal{A}_{n}\left(1^{a_{1}}, \ldots, n^{a_{n}}\right) \equiv\left\langle a_{1}, \ldots, a_{n} \mid \mathcal{M}_{n}\left(p_{1}, \ldots, p_{n}\right)\right\rangle
$$

where the $a_{i}$ are color indices. The divergences of $\mathcal{A}_{n}$ are encoded in the color operators $\boldsymbol{I}^{(1)}\left(\epsilon, \mu_{R}^{2} ;\{p\}\right)$ and $\boldsymbol{I}^{(2)}\left(\epsilon, \mu_{R}^{2} ;\{p\}\right)$. For the all-plus helicity case, the tree amplitude vanishes so $\boldsymbol{I}^{(2)}\left(\epsilon, \mu_{R}^{2} ;\{p\}\right)$ does not enter. The operator $\boldsymbol{I}^{(1)}\left(\epsilon, \mu_{R}^{2} ;\{p\}\right)$ is given by

$$
\boldsymbol{I}^{(1)}\left(\epsilon, \mu_{R}^{2} ;\{p\}\right)=\frac{1}{2} \frac{2}{(4 \pi)^{2-\epsilon}} \frac{1}{\Gamma(1-\epsilon)} \sum_{i=1}^{n} \sum_{j \neq i}^{n} \boldsymbol{T}_{i} \cdot \boldsymbol{T}_{j}\left[\frac{1}{\epsilon^{2}}\left(\frac{\mu_{R}^{2} e^{-i \lambda_{i j} \pi}}{2 p_{i} \cdot p_{j}}\right)^{\epsilon}+\frac{\gamma_{i}}{\boldsymbol{T}_{i}^{2}} \frac{1}{\epsilon}\right],
$$

where $\lambda_{i j}=+1$ if $i$ and $j$ are both incoming or outgoing partons and $\lambda_{i j}=0$ otherwise. The color charge $\boldsymbol{T}_{i}=\left\{T_{i}^{a}\right\}$ is a vector with respect to the generator label $a$, and an $S U\left(N_{c}\right)$ matrix with respect to the color indices of parton $i$. For the adjoint representation $T_{c b}^{a}=i f^{c a b}$, and so $\boldsymbol{T}_{i}^{2}=C_{A}=N_{c}$. Also, for external gluons in a pure glue theory, $\gamma_{g}=\frac{11}{6} C_{A}$. (We have not included an overall factor of $e^{-\epsilon \psi(1)}$ appearing in Catani's expression, but have included a factor of $2 /(4 \pi)^{2-\epsilon}$ because of differing overall normalization conventions.)

Specializing $\boldsymbol{I}^{(1)}\left(\epsilon, \mu_{R}^{2} ;\{p\}\right)$ to the pure gluon case, and then rewriting the divergent terms in eq. (5.1) in our notation gives,

$$
\left.\mathcal{A}_{n}^{2 \text {-loop, ren. }}\left(1^{a_{1}}, 2^{a_{2}}, \ldots, n^{a_{n}}\right)\right|_{\text {div. }}=\left.\sum_{i<j}^{n} \mathcal{A}_{n}^{(i, j), 2 \text {-loop, ren. }}(1,2,3, \ldots, n)\right|_{\text {div. }}
$$

where

$$
\begin{aligned}
& \left.\mathcal{A}_{n}^{(i, j), 2 \text {-loop, ren. }}(1,2,3, \ldots, n)\right|_{\text {div. }} \equiv-g^{2} c_{\Gamma} \tilde{f}^{b_{i} a_{i} c} \tilde{f}^{c a_{j} b_{j}}\left[\frac{1}{\epsilon^{2}}\left(-s_{i j}\right)^{-\epsilon}+\frac{11}{6} \frac{1}{\epsilon}\right] \\
& \times \mathcal{A}_{n}^{1-\mathrm{loop}}\left(1^{a_{1}}, 2^{a_{2}}, \ldots, i^{b_{i}}, \ldots, j^{b_{j}}, \ldots, n^{a_{n}}\right),
\end{aligned}
$$

and $\tilde{f}^{a b c}$ is defined in eq. (4.4). In converting the overall normalization we used

$$
\begin{aligned}
c_{\Gamma} & \equiv \frac{1}{(4 \pi)^{2-\epsilon}} \frac{\Gamma(1+\epsilon) \Gamma^{2}(1-\epsilon)}{\Gamma(1-2 \epsilon)} \\
& =\frac{1}{(4 \pi)^{2-\epsilon}} \frac{1}{\Gamma(1-\epsilon)}+\mathcal{O}\left(\epsilon^{3}\right) .
\end{aligned}
$$

Equation (5.4) holds for every pure gluon helicity amplitude of the form $\mathcal{A}_{n}( \pm,+,+, \ldots,+)$; such amplitudes vanish at tree-level due to supersymmetry Ward identities [11]. (Although the pure gluon theory is not supersymmetric, its tree amplitudes obey supersymmetry identities because the 
gluons' fermionic superpartners cannot contribute at tree level.) Specializing now to the four-point case, we have

$$
\left.\left.\mathcal{A}_{4}^{2 \text {-loop, ren. }}(1,2,3,4)\right|_{\text {div. }} \equiv \sum_{i<j}^{4} \mathcal{A}_{4}^{(i, j), 2 \text {-loop, ren. }}(1,2,3,4)\right|_{\text {div. }} .
$$

We shall evaluate the term in eq. (5.7) with $(i, j)=(1,2)$,

$$
\begin{aligned}
& \left.\mathcal{A}_{4}^{(1,2), 2 \text {-loop, ren. }}\left(1^{a_{1}}, 2^{a_{2}}, 3^{a_{3}}, 4^{a_{3}}\right)\right|_{\text {div. }}=-g^{2} c_{\Gamma}\left[\frac{1}{\epsilon^{2}}\left(-s_{12}\right)^{-\epsilon}+\frac{11}{6} \frac{1}{\epsilon}\right] \tilde{f}^{b_{1} a_{1} c} \tilde{f}^{c a_{2} b_{2}} \\
& \times \mathcal{A}_{4}^{1 \text {-loop }}\left(1^{b_{1}}, 2^{b_{2}}, 3^{a_{3}}, 4^{a_{4}}\right),
\end{aligned}
$$

and then obtain the other five cases by relabeling $i$ and $j$. Note that we count $(i, j)=(3,4)$ as being distinct from $(i, j)=(1,2)$ in the sum $(5.7)$.

The one-loop amplitudes may be decomposed in terms of $S U\left(N_{c}\right)$ structure constants [20] (see also eq. (2.30) of ref. [9]),

$$
\begin{gathered}
\mathcal{A}_{4}^{1 \text {-loop }}(1,2,3,4)=g^{4}\left[C_{1234}^{1 \text {-loop }} A_{4}^{1 \text {-loop }}(1,2,3,4)+C_{1243}^{1 \text {-loop }} A_{4}^{1 \text {-loop }}(1,2,4,3)\right. \\
\left.+C_{1423}^{1 \text {-loop }} A_{4}^{1 \text {-loop }}(1,4,2,3)\right]
\end{gathered}
$$

where $C_{1234}^{1-\text { loop }}$ is the color factor obtained by dressing each vertex in the one-loop box diagram in fig. 11 with a structure constant $\tilde{f}^{a b c}$, and dressing each bond between vertices with a $\delta^{a b}$. In this decomposition the $A_{4}^{1 \text {-loop }}$ are free of all group theory factors, and are invariant under cyclic permutations of their four arguments.

For the all-plus helicity case, the primitive amplitudes $A_{4}^{1 \text {-loop }}$ are proportional to a one-loop box integral [21],

$$
A_{4}^{1-\text { loop }}\left(1^{+}, 2^{+}, 3^{+}, 4^{+}\right)=\left(D_{s}-2\right) \frac{[12][34]}{\langle 12\rangle\langle 34\rangle} \mathcal{I}_{4}^{1-\text { loop }}\left[\lambda_{p}^{4}\right]\left(s_{12}, s_{23}\right)
$$

with

$$
\mathcal{I}_{4}^{1 \text {-loop }}\left[\lambda_{p}^{4}\right]\left(s_{12}, s_{23}\right) \equiv \int \frac{d^{D} p}{(2 \pi)^{4}} \frac{\lambda_{p}^{4}}{p^{2}\left(p-k_{1}\right)^{2}\left(p-k_{1}-k_{2}\right)^{2}\left(p+k_{4}\right)^{2}}
$$

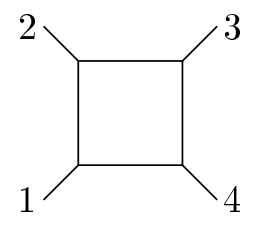

Figure 11: $C_{1234}^{1-\text { loop }}$ is given by dressing each vertex in the figure with $\tilde{f}^{a b c}$, and dressing each bond between vertices with a $\delta^{a b}$.

Using eq. (5.9) the divergence (5.8) is

$$
\begin{aligned}
\left.\mathcal{A}_{4}^{(1,2), 2 \text {-loop, ren. }}(1,2,3,4)\right|_{\text {div. }}=-g^{6} c_{\Gamma}\left[\frac{1}{\epsilon^{2}}(-\right. & \left.\left.s_{12}\right)^{-\epsilon}+\frac{11}{6} \frac{1}{\epsilon}\right] \tilde{f}^{b_{1} a_{1} c} \tilde{f}^{c a_{2} b_{2}}\left(C_{b_{1} b_{2} 34}^{1-\text { loop }} A_{4}^{1-\text { loop }}(1,2,3,4)\right. \\
& \left.+C_{b_{1} b_{2} 43}^{1-\text { loop }} A_{4}^{1-\text { loop }}(1,2,4,3)+C_{b_{1} 4 b_{2} 3}^{1 \text {-loop }} A_{4}^{1-\text { loop }}(1,4,2,3)\right) .
\end{aligned}
$$




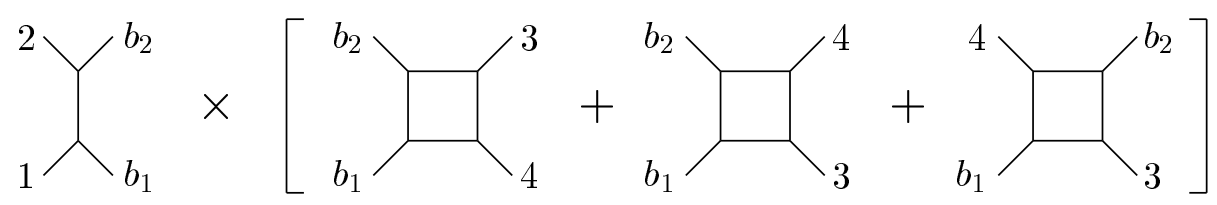

Figure 12: A schematic version of the color factors in eq. (5.15). The color factors are given by dressing each vertex with $\tilde{f}^{a b c}$, and dressing each bond between vertices with a $\delta^{a b}$.

We display a schematic version of the above color factors in fig. 12. Anticipating our comparison to the divergences appearing in the two-loop amplitude (4.3), we observe that the one-loop color coefficients can be related to the two-loop ones via,

$$
\begin{aligned}
& \tilde{f}^{b_{1} a_{1} c} \tilde{f}^{c a_{2} b_{2}} C_{b_{1} b_{2} 34}^{1-\text { lop }}=C_{1234}^{\mathrm{P}}, \\
& \tilde{f}^{b_{1} a_{1} c} \tilde{f}^{c a_{2} b_{2}} C_{b_{1} b_{2} 43}^{1-\text { lop }}=C_{1243}^{\mathrm{P}}, \\
& \tilde{f}^{b_{1} a_{1} c} \tilde{f}^{c a_{2} b_{2}} C_{b_{1} 4 b_{2} 3}^{1-\text { loop }}=C_{34 ; 12}^{\mathrm{NP}} .
\end{aligned}
$$

Using these relations in eq. (5.8) we have

$$
\begin{aligned}
& \left.\mathcal{A}_{4}^{(1,2), 2 \text {-loop, ren. }}(1,2,3,4)\right|_{\text {div. }}=-g^{6} c_{\Gamma}\left[\frac{1}{\epsilon^{2}}\left(-s_{12}\right)^{-\epsilon}+\frac{11}{6} \frac{1}{\epsilon}\right]\left(C_{1234}^{\mathrm{P}} A_{4}^{1-\text { loop }}(1,2,3,4)\right. \\
& \left.+C_{1243}^{\mathrm{P}} A_{4}^{1 \text {-loop }}(1,2,4,3)+C_{34 ; 12}^{\mathrm{NP}} A_{4}^{1 \text {-loop }}(1,4,2,3)\right) \text {, }
\end{aligned}
$$

so the color factors are the same ones appearing in the two-loop amplitude. Inserting eq. (5.14) into eq. (5.7), summing over $i$ and $j$, and making use of eq. (4.5), we obtain the predicted divergence,

$$
\begin{aligned}
& \left.\mathcal{A}_{4}^{2 \text {-loop, ren. }}(1,2,3,4)\right|_{\text {div. }} \\
& =-g^{6} c_{\Gamma}\left[\frac{1}{\epsilon^{2}}\left(-s_{12}\right)^{-\epsilon}+\frac{11}{6} \frac{1}{\epsilon}\right] \\
& \quad\left(2 C_{1234}^{\mathrm{P}} A_{4}^{1-\text { loop }}(1,2,3,4)+2 C_{3421}^{\mathrm{P}} A_{4}^{1-\text { loop }}(3,4,2,1)\right. \\
& \left.+C_{12 ; 34}^{\mathrm{NP}} A_{4}^{1-\text { loop }}(1,3,2,4)+C_{34 ; 21}^{\mathrm{NP}} A_{4}^{1-\text { loop }}(3,2,4,1)\right)+\mathcal{C}(234) .
\end{aligned}
$$

Now we compare eq. (5.15) to the divergences found in the two-loop all-plus helicity amplitude (4.3), using results for the two-loop integrals given in the appendix. All of the integrals are finite except for $\mathcal{I}_{4}^{\mathrm{P}}\left[\lambda_{p+q}^{2} \lambda_{q}^{2}\right], \mathcal{I}_{4}^{\mathrm{P}}\left[\lambda_{p+q}^{2} \lambda_{p}^{2}\right]$ and $\mathcal{I}_{4}^{\mathrm{NP}}\left[\lambda_{p}^{2} \lambda_{q}^{2}\right]$. For eq. (5.15) to be recovered, it is crucial that the divergent parts of these integrals are proportional to the same one-loop box integral (5.11) encountered in $A_{4}^{1 \text {-loop }}$

$$
\begin{aligned}
\left.\mathcal{I}_{4}^{\mathrm{P}}\left[\lambda_{p+q}^{2} \lambda_{q}^{2}\right]\left(s_{12}, s_{23}\right)\right|_{\text {div. }}= & \left.\mathcal{I}_{4}^{\mathrm{P}}\left[\lambda_{p+q}^{2} \lambda_{p}^{2}\right]\left(s_{12}, s_{23}\right)\right|_{\text {div. }}=-i c_{\Gamma} \frac{1}{\epsilon^{2}}\left(-s_{12}\right)^{-1-\epsilon} \mathcal{I}_{4}^{1-\text { loop }}\left[\lambda_{p}^{4}\right]\left(s_{12}, s_{23}\right) \\
& \left.\mathcal{I}_{4}^{\mathrm{NP}}\left[\lambda_{p}^{2} \lambda_{q}^{2}\right]\left(s_{12}, s_{23}\right)\right|_{\text {div. }}=-i c_{\Gamma} \frac{1}{\epsilon^{2}}\left(-s_{12}\right)^{-1-\epsilon} \mathcal{I}_{4}^{1-\text { loop }}\left[\lambda_{p}^{4}\right]\left(s_{13}, s_{23}\right)
\end{aligned}
$$

as can be seen from eqs. (A.11) and (A.14).

This factorization of the singular behavior of the integrals can be understood heuristically. The $1 / \epsilon^{2}$ poles come from loop momenta that are simultaneously soft and collinear with two adjacent 
external legs, so that three consecutive propagators can go on shell. Because the external momenta are strictly four-dimensional, the singular region of loop momenta is also four-dimensional. However, a factor of $\lambda_{p}^{2}$ in the numerator suppresses the region where $p$ becomes four-dimensional. Thus in $\mathcal{I}_{4}^{\mathrm{P}}\left[\lambda_{p+q}^{2} \lambda_{q}^{2}\right]$, for example, the region with $\lambda_{p}^{2} \approx 0$ and $p^{2} \approx 0$ dominates the integral, while $q$ and $p+q$ remain off shell. The space-time picture of the dominant integration region corresponds to shrinking the rightmost box of the planar double box in fig. 5(a) to a small size. The kinematics of that inner box integral is hardly affected by the soft momentum $p$, so it is essentially the same as the external kinematics, and it can be factored out of the outer triangle integral that remains. The triangle integral gives the factor of $-i c_{\Gamma}\left(-s_{12}\right)^{-1-\epsilon} / \epsilon^{2}$ 'dressing' the box integral.

Apart from those in eq. (5.16), all the other integrals appearing in the two-loop all-plus amplitude are finite in the limit $\epsilon \rightarrow 0$. For these integrals, whenever there is a loop momentum from the set $\{p, q, p+q\}$ whose four-dimensional limit is not suppressed by a numerator factor of $\lambda_{i}^{2}$, then that loop momentum has at most two propagators containing it, not three. This property prevents an infrared divergence from occurring.

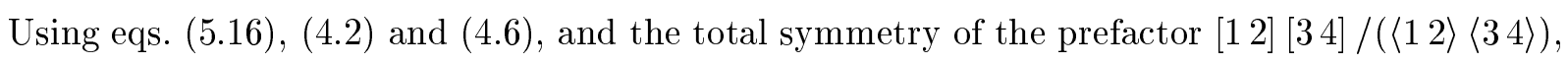
the divergences of the bare (unrenormalized) primitive amplitudes are

$$
\begin{aligned}
& \left.A_{1234}^{\mathrm{P}}\right|_{\text {div. }}=-2 c_{\Gamma} \frac{1}{\epsilon^{2}}\left(-s_{12}\right)^{-\epsilon} A_{4}^{1-\mathrm{loop}}\left(1^{+}, 2^{+}, 3^{+}, 4^{+}\right), \\
& \left.A_{12 ; 34}^{\mathrm{NP}}\right|_{\text {div. }}=-c_{\Gamma} \frac{1}{\epsilon^{2}}\left(-s_{12}\right)^{-\epsilon} A_{4}^{1-\mathrm{loop}}\left(1^{+}, 3^{+}, 2^{+}, 4^{+}\right) .
\end{aligned}
$$

Inserting these expressions into the bare full amplitude (4.3) then yields

$$
\begin{gathered}
\left.\mathcal{A}_{4}^{2-\text { loop }}(1,2,3,4)\right|_{\text {div. }}=-g^{6} c_{\Gamma}\left[\frac{2}{\epsilon^{2}}\left(-s_{12}\right)^{-\epsilon} C_{1234}^{\mathrm{P}} A_{4}^{1-\text { loop }}(1,2,3,4)+\frac{2}{\epsilon^{2}}\left(-s_{12}\right)^{-\epsilon} C_{3421}^{\mathrm{P}} A_{4}^{1-\text { loop }}(3,4,2,1)\right. \\
\left.+\frac{1}{\epsilon^{2}}\left(-s_{12}\right)^{-\epsilon} C_{12 ; 34}^{\mathrm{NP}} A_{4}^{1-\text { loop }}(1,3,2,4)+\frac{1}{\epsilon^{2}}\left(-s_{12}\right)^{-\epsilon} C_{34 ; 21}^{\mathrm{NP}} A_{4}^{1-\mathrm{loop}}(3,2,4,1)\right] \\
+\mathcal{C}(234) .
\end{gathered}
$$

The expression (5.18) already agrees with eq. (5.15) at the level of the $\left(-s_{i j}\right)^{-\epsilon} / \epsilon^{2}$ poles. To check the agreement at the $1 / \epsilon$ level, we must first renormalize our two-loop amplitude in the $\overline{\mathrm{MS}}$ scheme. The $\overline{\mathrm{MS}}$ counterterm to be subtracted from $\mathcal{A}_{4}^{2-\text { loop }}(1,2,3,4)$ is

$$
\begin{aligned}
\text { C.T. } & =4 g^{2} c_{\Gamma} \frac{11 N_{c}}{6} \frac{1}{\epsilon} \mathcal{A}_{4}^{1-\text { loop }}\left(1^{+}, 2^{+}, 3^{+}, 4^{+}\right), \\
& =4 N_{c} g^{6} c_{\Gamma} \frac{11}{6} \frac{1}{\epsilon}\left[C_{1234}^{1-\text { loop }} A_{4}^{1-\text { loop }}(1,2,3,4)+C_{3124}^{1 \text {-loop }} A_{4}^{1 \text {-loop }}(3,1,2,4)+C_{2314}^{1 \text {-loop }} A_{4}^{1 \text {-loop }}(2,3,1,4)\right] .
\end{aligned}
$$

The relative simplicity of the ultraviolet subtraction term is due to the vanishing of the corresponding tree-level helicity amplitude; the counterterm has the same structure encountered in other helicity configurations at one loop, up to the overall factor and the replacement of a tree amplitude with the one-loop amplitude. (At two loops, an additional $1 / \epsilon^{2}$ subtraction is required for helicity configurations with non-vanishing tree amplitudes. Additional subtraction terms are necessary for amplitudes including fermions or scalars as well.) 
Finally, we use the color conservation identity $\sum_{i} \boldsymbol{T}_{i}=0$ [18], which implies that

$$
n N_{c}\left|\mathcal{M}_{n}\right\rangle=\sum_{i=1}^{n} \boldsymbol{T}_{i}^{2}\left|\mathcal{M}_{n}\right\rangle=-\sum_{j \neq i}^{n} \boldsymbol{T}_{i} \cdot \boldsymbol{T}_{j}\left|\mathcal{M}_{n}\right\rangle
$$

when all external particles are in the adjoint representation. Replacing the $4 N_{c}$ in eq. (5.19) with the help of eq. (5.20), the color structure of the $\overline{\mathrm{MS}}$ counterterm (and its dependence on $A_{4}^{1-\text { loop }}$ ) can be put in exactly the same form as Catani's expression for the divergent terms, eqs. (5.1), (5.3) and (5.4). We can then process it into the form (5.15) in just the same way, obtaining

$$
\begin{aligned}
\text { C.T. }=g^{6} c_{\Gamma} & {\left[\frac{11}{3} \frac{1}{\epsilon} C_{1234}^{\mathrm{P}} A_{4}^{1-\mathrm{loop}}(1,2,3,4)+\frac{11}{3} \frac{1}{\epsilon} C_{3421}^{\mathrm{P}} A_{4}^{1-\mathrm{loop}}(3,4,2,1)\right.} \\
+ & \left.\frac{11}{6} \frac{1}{\epsilon} C_{12 ; 34}^{\mathrm{NP}} A_{4}^{1-\mathrm{loop}}(1,3,2,4)+\frac{11}{6} \frac{1}{\epsilon} C_{34 ; 21}^{\mathrm{NP}} A_{4}^{1-\mathrm{loop}}(3,2,4,1)\right]+\mathcal{C}(234) .
\end{aligned}
$$

After including the ultraviolet subtraction (5.21), the infrared divergences of our renormalized amplitude agree perfectly with the expected form (5.15).

\section{Conclusions}

In this paper we presented the pure glue contribution to the identical helicity two-loop four-gluon amplitude in QCD. We calculated the amplitude using its analytic properties in $D$ dimensions. More generally, any amplitude in a massless theory can in principle be obtained in this way. Although QCD is not supersymmetric, we also used supersymmetry as a helpful guide in constructing the pure gluon amplitude from amplitudes with scalar loops. Cases with quarks will be discussed elsewhere.

We expect the type of methods used here to be useful for obtaining more general two-loop amplitudes relevant for high energy processes. Although much more remains to be done, we are hopeful that calculations of next-to-next-to-leading-order multi-jet cross sections will become possible.

\section{Acknowledgments}

We thank R. Blankenbecler, H. Haber, K. Melnikov and M. Peskin for useful discussions regarding two-loop integrals.

\section{A Integrals}

In this appendix we evaluate the integrals appearing in the all-plus helicity amplitude, in an expansion in $\epsilon$ through $\mathcal{O}\left(\epsilon^{0}\right)$.

The planar bow-tie integrals are rather simple to evaluate because they are products of one-loop integrals,

$$
\begin{aligned}
\mathcal{I}_{4}^{\text {bow-tie }}\left[\lambda_{p}^{2} \lambda_{q}^{2}\right](s) & =-\frac{1}{4} \frac{1}{(4 \pi)^{4}}, \\
\mathcal{I}_{4}^{\text {bow-tie }}\left[\lambda_{p}^{2} \lambda_{q}^{2}(p+q)^{2}\right](s, t) & =-\frac{1}{36} \frac{1}{(4 \pi)^{4}}(t-4 s), \\
\mathcal{I}_{4}^{\text {bow-tie }}\left[\lambda_{p}^{2}\left(\lambda_{p} \cdot \lambda_{q}\right)\right](s) & =0 .
\end{aligned}
$$


Two of the planar double box integrals and one of the non-planar ones vanish through $\mathcal{O}\left(\epsilon^{0}\right)$,

$$
\begin{aligned}
\mathcal{I}_{4}^{\mathrm{P}}\left[\left(\lambda_{p} \cdot \lambda_{q}\right)^{2}\right](s, t) & =0, \\
\mathcal{I}_{4}^{\mathrm{P}}\left[\lambda_{p}^{2} \lambda_{q}^{2}\right](s, t) & =0, \\
\mathcal{I}_{4}^{\mathrm{NP}}\left[\left(\lambda_{p} \cdot \lambda_{q}\right)^{2}-\lambda_{p}^{2} \lambda_{q}^{2}\right](s, t) & =0 .
\end{aligned}
$$

The non-vanishing planar double box integral, evaluated in its Euclidean region where $s, t<0$ and $u>0$, is,

$$
\begin{aligned}
& \mathcal{I}_{4}^{\mathrm{P}}\left[\lambda_{p+q}^{2} \lambda_{q}^{2}\right](s, t)=\mathcal{I}_{4}^{\mathrm{P}}\left[\lambda_{p+q}^{2} \lambda_{p}^{2}\right](s, t) \\
& =c_{2 \Gamma}(-s)^{-1-2 \epsilon} \frac{1}{6}\left\{-\frac{1}{\epsilon^{2}}+\frac{1}{\epsilon}\left[\frac{1}{2} \frac{\chi\left(\ln ^{2} \chi+\pi^{2}\right)}{(1+\chi)^{2}}+\frac{\chi \ln \chi}{1+\chi}-\frac{8}{3}\right]\right. \\
& \quad+\frac{\chi}{(1+\chi)^{2}}\left[-\operatorname{Li}_{3}(-\chi)+\zeta(3)+\ln \chi \operatorname{Li}_{2}(-\chi)+\left(\frac{1}{2} \ln (1+\chi)-\frac{2}{3} \ln \chi-\frac{1}{2} \chi+\frac{1}{3}\right)\left(\ln ^{2} \chi+\pi^{2}\right)\right. \\
& \left.\left.\quad+\left(\frac{8}{3}(1+\chi)-\frac{\pi^{2}}{6}\right) \ln \chi\right]-\frac{\pi^{2}}{6} \frac{2-5 \chi}{1+\chi}-\frac{52}{9}\right\}
\end{aligned}
$$

where $\chi=t / s$ and

$$
c_{2 \Gamma} \equiv \frac{1}{(4 \pi)^{4-2 \epsilon}} \frac{\Gamma(1+2 \epsilon) \Gamma^{3}(1-\epsilon)}{\Gamma(1-3 \epsilon)} .
$$

The polylogarithms are [22]

$$
\mathrm{Li}_{2}(x)=-\int_{0}^{x} d t \frac{\ln (1-t)}{t}, \quad \mathrm{Li}_{3}(x)=\int_{0}^{x} d t \frac{\mathrm{Li}_{2}(t)}{t}
$$

and $\zeta(3)=\mathrm{Li}_{3}(1)=1.202 \ldots$ Equation (A.2) was originally found by direct integration over Feynman parameters, after performing certain subtractions to allow expansions of integrands in $\epsilon$. We have also checked that the same result can be obtained via the general tensor integral reduction method of Smirnov and Veretin [5].

The remaining planar integrals in the full amplitude (4.3) may be obtained from eq. (A.2) by relabeling the legs, which induces transformations on $\chi$, for example,

$$
\begin{array}{lll}
s \leftrightarrow t & \Leftrightarrow & \chi \leftrightarrow \frac{1}{\chi}, \\
t \leftrightarrow u & \Leftrightarrow & \chi \leftrightarrow-1-\chi, \\
s \leftrightarrow u & \Leftrightarrow & \chi \leftrightarrow \frac{-\chi}{1+\chi},
\end{array}
$$

using $s+t+u=0$.

For two reasons, it is useful to represent the poles in $\epsilon$ of eq. (A.2), and of the non-planar integral $\mathcal{I}_{4}^{\mathrm{NP}}\left[\lambda_{p}^{2} \lambda_{q}^{2}\right]$, in terms of the one-loop box integral $\mathcal{I}_{4}^{1-\mathrm{loop}}\left[\lambda_{p}^{4}\right]$ defined in eq. (5.11). First, the divergence structure of the two-loop all-plus helicity amplitude becomes more transparent, as discussed in section 5, given that $\mathcal{I}_{4}^{1 \text {-loop }}\left[\lambda_{p}^{4}\right]$ appears in the corresponding one-loop amplitude. Second, the description of the finite $\left(\mathcal{O}\left(\epsilon^{0}\right)\right)$ remainder terms is simplified, especially regarding their values in different kinematic regions. The heuristic reason why the singular parts of the two-loop integrals are proportional to $\mathcal{I}_{4}^{1 \text {-loop }}\left[\lambda_{p}^{4}\right]$ was given in section 5 . 
The one-loop box integral (5.11) can be performed by first integrating over the $(-2 \epsilon)$-dimensional components of $p$, which results in an integral of the scalar type but in $8-2 \epsilon$ dimensions, not $4-2 \epsilon$. A standard one-loop 'dimension-shifting' identity allows one to trade this integral for a quantity $\mathcal{L}(s, t)$ which is essentially the scalar integral in $6-2 \epsilon$ dimensions [23]. This integral has neither ultraviolet nor infrared divergences, which makes it straightforward to expand in $\epsilon$.

Concretely, we have

$$
\begin{aligned}
\mathcal{I}_{4}^{1-\text { loop }}\left[\lambda_{p}^{4}\right](s, t) & =-\epsilon(1-\epsilon) \mathcal{I}_{4}^{1-\text { loop }, D=8-2 \epsilon}[1](s, t) \\
& =\frac{-\epsilon(1-\epsilon)}{(1-2 \epsilon)(3-2 \epsilon)}\left[-\frac{1}{4} \frac{\chi \mathcal{L}(s, t)}{(1+\chi)^{2}}+\frac{1}{2} \frac{i c_{\Gamma}(-s)^{-\epsilon}}{\epsilon(1-\epsilon)} \frac{1+\chi^{1-\epsilon}}{1+\chi}\right]
\end{aligned}
$$

where $\chi=t / s$. The prefactor $c_{\Gamma}$ is defined in eq. (5.6), and

$$
\begin{aligned}
\mathcal{L}(s, t) & \equiv 2(1-2 \epsilon)(-s)(1+\chi) \mathcal{I}_{4}^{1-\text { loop, } D=6-2 \epsilon}[1](s, t) \\
& =2 i c_{\Gamma}(-s)^{-\epsilon}(1+\chi) \int_{0}^{1} d x \frac{1}{\epsilon} \frac{\chi^{-\epsilon} x^{-\epsilon}-(1-x)^{-\epsilon}}{1-(1+\chi) x} \\
& =2 i c_{\Gamma}(-s)^{-\epsilon}(1+\chi) \int_{0}^{1} d x \frac{1}{1-(1+\chi) x}\left[\ln \left(\frac{1-x}{\chi x}\right)-\frac{\epsilon}{2}\left(\ln ^{2}(1-x)-\ln ^{2}(\chi x)\right)+\mathcal{O}\left(\epsilon^{2}\right)\right] .
\end{aligned}
$$

Performing the one-dimensional integral we get,

$$
\begin{aligned}
& \mathcal{L}(s, t)=i c_{\Gamma}(-s)^{-\epsilon}\left\{\ln ^{2} \chi+\pi^{2}\right. \\
& \left.+\epsilon\left[2\left(-\operatorname{Li}_{3}(-\chi)+\zeta(3)+\ln \chi \operatorname{Li}_{2}(-\chi)\right)+\left(\ln ^{2} \chi+\pi^{2}\right) \ln (1+\chi)-\frac{2}{3} \ln ^{3} \chi-\pi^{2} \ln \chi\right]\right\} \\
& \quad+\mathcal{O}\left(\epsilon^{2}\right) .
\end{aligned}
$$

Using eqs. (A.6) and (A.8), the desired one-loop integral is

$$
\begin{gathered}
\mathcal{I}_{4}^{1-\text { loop }}\left[\lambda_{p}^{4}\right](s, t)=i c_{\Gamma}(-s)^{-\epsilon}(-\epsilon)(1-\epsilon) \frac{1}{6}\left\{\frac{1}{\epsilon}-\frac{1}{2} \frac{\chi\left(\ln ^{2} \chi+\pi^{2}\right)}{(1+\chi)^{2}}-\frac{\chi \ln \chi}{1+\chi}+\frac{11}{3}\right. \\
+\epsilon\left[\frac { \chi } { ( 1 + \chi ) ^ { 2 } } \left[\operatorname{Li}_{3}(-\chi)-\zeta(3)-\ln \chi \operatorname{Li}_{2}(-\chi)+\frac{1}{3} \ln ^{3} \chi-\frac{1}{2} \ln ^{2} \chi \ln (1+\chi)\right.\right. \\
\left.\quad+\frac{\pi^{2}}{2} \ln \left(\frac{\chi}{1+\chi}\right)+\frac{1}{2}\left((2+\chi) \ln ^{2} \chi+\pi^{2}\right)\right] \\
\left.\left.+\frac{11}{3}\left(-\frac{1}{2} \frac{\chi\left(\ln ^{2} \chi+\pi^{2}\right)}{(1+\chi)^{2}}-\frac{\chi \ln \chi}{1+\chi}+\frac{11}{3}\right)-4\right]\right\} \\
+\mathcal{O}\left(\epsilon^{3}\right), \quad \operatorname{region~(i).~}
\end{gathered}
$$

The three kinematic regions for the four-point amplitude are

(i) $u>0$ and $s, t<0$, for which $\chi>0$;

(ii) $s>0$ and $t, u<0$, for which $-1<\chi<0$;

(iii) $t>0$ and $s, u<0$, for which $\chi<-1$. 
The form (A.9) for the one-loop box integral is appropriate for region (i), where it is manifestly real. In regions (ii) and (iii) the integral acquires an imaginary part. A form appropriate for region (ii) is obtained by substituting $(-s)^{-\epsilon} \rightarrow s^{-\epsilon}\left(1+\epsilon i \pi-\epsilon^{2} \pi^{2} / 2\right)$ and $\ln \chi \rightarrow \ln |\chi|+i \pi$,

$$
\begin{aligned}
\mathcal{I}_{4}^{1-\operatorname{loop}}\left[\lambda_{p}^{4}\right](s, t)= & i c_{\Gamma} s^{-\epsilon}(-\epsilon)(1-\epsilon) \frac{1}{6}\left\{\frac{1}{\epsilon}-\frac{1}{2} \frac{\chi \ln ^{2}|\chi|}{(1+\chi)^{2}}-\frac{\chi \ln |\chi|}{1+\chi}+\frac{11}{3}\right. \\
+ & +\epsilon\left[\frac { \chi } { ( 1 + \chi ) ^ { 2 } } \left[\operatorname{Li}_{3}(-\chi)-\zeta(3)-\ln |\chi| \operatorname{Li}_{2}(-\chi)+\frac{1}{3} \ln ^{3}|\chi|-\frac{1}{2} \ln ^{2}|\chi| \ln (1+\chi)\right.\right. \\
& \left.+\frac{\pi^{2}}{2} \ln |\chi|+\frac{1}{2}(2+\chi) \ln ^{2}|\chi|\right] \\
+ & i \pi\left(-\frac{\pi^{2}}{2(1+\chi)}+\frac{11}{3}\left(-\frac{1}{2} \frac{\chi \ln ^{2}|\chi|}{(1+\chi)^{2}}+\frac{\chi \ln |\chi|}{1+\chi}+\frac{11}{3}\right)-4\right] \\
& \left.\left.+\epsilon\left[\frac{\chi}{(1+\chi)^{2}}\left[\operatorname{Li}_{2}(1+\chi)+\frac{1}{2} \ln ^{2}|\chi|+\ln |\chi|\right]-\frac{11}{3}\left(\frac{\chi \ln |\chi|}{(1+\chi)^{2}}-\frac{1}{1+\chi}\right)\right]\right)\right\} \\
+ & \mathcal{O}\left(\epsilon^{3}\right), \quad \operatorname{region}(\text { ii }) .
\end{aligned}
$$

A form appropriate for region (iii) can be obtained simply by applying the transformation $s \leftrightarrow t$ $(\chi \leftrightarrow 1 / \chi)$ to eq. (A.10), since that transformation maps region (ii) into region (iii).

We now return to the non-vanishing planar double box integral. Its expression in terms of $\mathcal{I}_{4}^{1-\text { loop }}\left[\lambda_{p}^{4}\right]$ is,

$$
\mathcal{I}_{4}^{\mathrm{P}}\left[\lambda_{p+q}^{2} \lambda_{q}^{2}\right](s, t)=-i c_{\Gamma} \frac{1}{\epsilon^{2}}(-s)^{-1-\epsilon} \mathcal{I}_{4}^{1-\mathrm{loop}}\left[\lambda_{p}^{4}\right](s, t)+\frac{F_{p+q, q}^{\mathrm{P}}}{(4 \pi)^{4}(-s)}+\mathcal{O}(\epsilon),
$$

where the finite remainder is

$$
\begin{array}{rlrl}
F_{p+q, q}^{\mathrm{P}} & =\frac{1}{18} \frac{\chi}{(1+\chi)^{2}}\left[-\ln \chi\left(\ln ^{2} \chi+\pi^{2}\right)+\left(\chi-\frac{1}{\chi}\right) \pi^{2}\right], & & \text { region (i), } \\
& =\frac{1}{18} \frac{\chi}{(1+\chi)^{2}}\left[-\ln |\chi|\left(\ln ^{2}|\chi|-2 \pi^{2}\right)+\left(\chi-\frac{1}{\chi}\right) \pi^{2}-3 i \pi \ln ^{2}|\chi|\right], & \text { regions (ii) and (iii). }
\end{array}
$$

In rewriting eq. (A.2), we used

$$
c_{2 \Gamma}=c_{\Gamma}^{2}\left[1+\mathcal{O}\left(\epsilon^{3}\right)\right] .
$$

We remark that the apparent power-law singularities as $\chi \rightarrow-1$ in eqs. (A.10) and (A.12) are all spurious, cancelling among the various terms.

The analytic structure of the non-planar integrals is more intricate than that of the planar ones. This is because, for Mandelstam variables $s, t, u$ satisfying $s+t+u=0$, there is no Euclidean region where the integrals are purely real. (It is possible to find a Euclidean region by relaxing the condition $s+t+u=0$, but this introduces another dimensionless variable and leads to more cumbersome expressions [6].) Also in contrast to the planar case, the non-planar integrals do not have a uniform $i \varepsilon$ prescription in terms of the dimensionless ratio $\chi$. The correct $i \varepsilon$ prescription can be determined by following through the $i \varepsilon s$ appearing in the Feynman propagators. 
We express the divergent non-planar integral in terms of the one-loop box integral, as in eq. (A.11),

$$
\mathcal{I}_{4}^{\mathrm{NP}}\left[\lambda_{p}^{2} \lambda_{q}^{2}\right](s, t)=-i c_{\Gamma} \frac{1}{\epsilon^{2}}(-s)^{-1-\epsilon} \mathcal{I}_{4}^{1-\mathrm{loop}}\left[\lambda_{p}^{4}\right](u, t)+\frac{F_{p, q}^{\mathrm{NP}}}{(4 \pi)^{4}(-s)}+\mathcal{O}(\epsilon)
$$

Here the finite remainder is

$$
\begin{aligned}
F_{p, q}^{\mathrm{NP}}= & \frac{1}{6}\left\{-2 \chi(1+\chi)\left[\operatorname{Li}_{3}\left(\frac{\chi}{1+\chi}\right)-\zeta(3)-\ln \left(\frac{\chi}{1+\chi}\right)\left(\operatorname{Li}_{2}\left(\frac{\chi}{1+\chi}\right)+\frac{\pi^{2}}{2}\right)-\frac{1}{6} \ln ^{3}\left(\frac{\chi}{1+\chi}\right)\right]\right. \\
& +3 \chi(1+\chi) \ln (1+\chi) \ln \chi-\frac{1}{2}(1+\chi)^{2}\left(-\frac{1}{\chi}+3\right) \ln ^{2}(1+\chi)-\frac{1}{2} \chi^{2}\left(\frac{1}{1+\chi}+3\right) \ln ^{2} \chi \\
& +\pi^{2}\left(\chi-\frac{1}{2} \frac{1}{1+\chi}+\frac{5}{6}\right)+(1+\chi) \ln (1+\chi)-\chi \ln \chi \\
+ & \left.i \pi\left(2 \chi(1+\chi)\left[\operatorname{Li}_{2}\left(\frac{\chi}{1+\chi}\right)-\frac{\pi^{2}}{6}-\frac{3}{2} \ln \chi\right]+(1+\chi)\left[(1+\chi)\left(-\frac{1}{\chi}+3\right) \ln (1+\chi)-1\right]\right)\right\}, \\
& \quad \text { region (i), }
\end{aligned}
$$

$$
\begin{aligned}
F_{p, q}^{\mathrm{NP}}= & \frac{1}{6}\left\{-2 \chi(1+\chi)\left[\operatorname{Li}_{3}\left(\frac{\chi}{1+\chi}\right)-\zeta(3)-\ln \left|\frac{\chi}{1+\chi}\right| \operatorname{Li}_{2}\left(\frac{\chi}{1+\chi}\right)-\frac{1}{6} \ln ^{3}\left|\frac{\chi}{1+\chi}\right|\right]\right. \\
& +3 \chi(1+\chi)\left(\ln (1+\chi) \ln |\chi|-\frac{\pi^{2}}{2}\right)-\frac{1}{2}(1+\chi)^{2}\left(-\frac{1}{\chi}+3\right) \ln ^{2}(1+\chi) \\
& -\frac{1}{2} \chi^{2}\left(\frac{1}{1+\chi}+3\right) \ln ^{2}|\chi|+\frac{\pi^{2}}{3}+(1+\chi) \ln (1+\chi)-\chi \ln |\chi| \\
+ & \left.i \pi\left(\chi(1+\chi)\left[\ln ^{2}\left|\frac{\chi}{1+\chi}\right|+\pi^{2}\right]-(1+\chi)\left(-\frac{1}{\chi}+2\right) \ln (1+\chi)+\chi\left(\frac{1}{1+\chi}+2\right) \ln |\chi|+1\right)\right\}, \\
& \quad \text { region (ii), }
\end{aligned}
$$

and the form for region (iii) may be obtained from that for region (i) through the transformation $t \leftrightarrow u(\chi \leftrightarrow-1-\chi)$. The region (ii) form for $F_{p, q}^{\mathrm{NP}}$ is symmetric under $\chi \leftrightarrow-1-\chi$, although the identities $[22]$

$$
\begin{array}{ll}
\operatorname{Li}_{3}(-x)-\mathrm{Li}_{3}(-1 / x)=-\frac{\pi^{2}}{6} \ln x-\frac{1}{6} \ln ^{3} x, & x>0 \\
\mathrm{Li}_{2}(-x)+\mathrm{Li}_{2}(-1 / x)=-\frac{\pi^{2}}{6}-\frac{1}{2} \ln ^{2} x, & x>0
\end{array}
$$

are needed to demonstrate this.

Finally, the integral $\mathcal{I}_{4}^{\mathrm{NP}}\left[\lambda_{p+q}^{2} \lambda_{q}^{2}\right]=\mathcal{I}_{4}^{\mathrm{NP}}\left[\lambda_{p+q}^{2} \lambda_{p}^{2}\right]$ is finite, but non-vanishing, as $\epsilon \rightarrow 0$. It is given 
by

$$
\begin{aligned}
\mathcal{I}_{4}^{\mathrm{NP}}\left[\lambda_{p+q}^{2} \lambda_{q}^{2}\right]= & \frac{1}{(4 \pi)^{4}(-s)} \frac{1}{6}\left\{\frac{\chi}{(1+\chi)^{2}}\left[\operatorname{Li}_{3}(-\chi)-\zeta(3)-\ln \chi\left(\operatorname{Li}_{2}(-\chi)-\frac{\pi^{2}}{6}\right)-\frac{3}{4} \chi\left(\ln ^{2} \chi-\pi^{2}\right)\right]\right. \\
& -\frac{1+\chi}{\chi^{2}}\left[\operatorname{Li}_{3}\left(\frac{1}{1+\chi}\right)-\zeta(3)+\ln (1+\chi)\left(\operatorname{Li}_{2}\left(\frac{1}{1+\chi}\right)+\frac{\pi^{2}}{6}\right)+\frac{3}{4}(1+\chi) \ln ^{2}(1+\chi)\right. \\
& \left.\quad+\frac{1}{3} \ln ^{3}(1+\chi)\right]+\left(\frac{1}{\chi(1+\chi)}+\frac{3}{2}\right) \ln (1+\chi) \ln \chi \\
& +\pi^{2}\left(\frac{1}{6 \chi}+\frac{4}{3(1+\chi)}+\frac{3}{2} \frac{\chi}{(1+\chi)^{2}}-\frac{3}{4}\right)+\frac{\ln (1+\chi)}{2 \chi}-\frac{\ln \chi}{2(1+\chi)} \\
+ & i \pi\left(-\frac{1+\chi}{\chi^{2}}\left[\operatorname{Li}_{2}\left(\frac{\chi}{1+\chi}\right)-\ln (1+\chi) \ln \chi+\frac{1}{2} \ln ^{2}(1+\chi)-\frac{3}{2}(1+\chi) \ln (1+\chi)\right]\right. \\
& \left.\left.-\frac{1}{2} \frac{\chi}{(1+\chi)^{2}}\left(\ln { }^{2} \chi+\pi^{2}\right)-\left(\frac{1}{\chi(1+\chi)}+\frac{3}{2}\right) \ln \chi-\frac{1}{2 \chi}\right)\right\}, \\
\mathcal{I}_{4}^{\mathrm{NP}}\left[\lambda_{p+q}^{2} \lambda_{q}^{2}\right]= & \frac{1}{(4 \pi)^{4}(-s)} \frac{1}{6}\left\{\frac{\chi}{(1+\chi)^{2}}\left[\operatorname{Li}(-\chi)-\zeta(3)-\ln |\chi|\left(\operatorname{Li}_{2}(-\chi)+\frac{5 \pi^{2}}{6}\right)-\frac{3}{4} \chi \ln ^{2}|\chi|\right]\right. \\
& +\frac{1}{2}\left(\frac{1}{\chi(1+\chi)}+\frac{3}{2}\right) \ln (1+\chi) \ln |\chi|-\pi^{2}\left(\frac{5}{12 \chi(1+\chi)}+\frac{3}{8}\right)-\frac{\ln |\chi|}{2(1+\chi)} \\
+ & i \pi\left(-\frac{\chi}{(1+\chi)^{2}}\left[\operatorname{Li}_{2}\left(\frac{1+\chi}{\chi}\right)-\ln (1+\chi) \ln |\chi|-\frac{3}{2} \ln |\chi|\right]\right. \\
& \left.\left.\quad \frac{1}{1+\chi}\left[\ln (1+\chi)-\frac{1}{2} \ln |\chi|+\frac{1}{2}\right]\right)+(\chi \leftrightarrow-1-\chi)\right\}, \\
& \operatorname{region}(\mathrm{ii}) .
\end{aligned}
$$

Again the form for region (iii) may be obtained from that for region (i) through the transformation $t \leftrightarrow u(\chi \leftrightarrow-1-\chi)$.

\section{References}

[1] M.L. Mangano and S.J. Parke, Phys. Rept. 200, 301 (1991);

L. Dixon, in Proceedings of Theoretical Advanced Study Institute in Elementary Particle Physics (TASI 95), ed. D.E. Soper [hep-ph/9601359].

[2] Z. Bern, L. Dixon and D.A. Kosower, Ann. Rev. Nucl. Part. Sci. 46, 109 (1996) [hep$\mathrm{ph} / 9602280]$.

[3] S.G. Gorishnii, A.L. Kataev and S.A. Larin, Phys. Lett. B259, 144 (1991);

L.R. Surguladze and M.A. Samuel, Phys. Rev. Lett. 66, 560 (1991), err. ibid. 66, 2416 (1991); T. van Ritbergen, J.A. Vermaseren and S.A. Larin, Phys. Lett. B400, 379 (1997) [hep$\mathrm{ph} / 9701390]$.

[4] J.M. Campbell and E.W.N. Glover, Nucl. Phys. B527, 264 (1998) [hep-ph/9710255];

S. Catani and M. Grazzini, Phys. Lett. B446, 143 (1999) [hep-ph/9810389]; hep-ph/9908523; 
Z. Bern, V. Del Duca and C.R. Schmidt, Phys. Lett. B445, 168 (1998) [hep-ph/9810409];

Z. Bern, V. Del Duca, W.B. Kilgore and C.R. Schmidt, Phys. Rev. D60, 116001 (1999) [hep$\mathrm{ph} / 9903516]$

D.A. Kosower and P. Uwer, hep-ph/9903515.

[5] V.A. Smirnov, Phys. Lett. B460, 397 (1999) [hep-ph/9905323];

V.A. Smirnov and O.L. Veretin, hep-ph/9907385.

[6] J.B. Tausk, hep-ph/9909506. C. Anastasiou, E.W.N. Glover and C. Oleari, hep-ph/9907523; hep-ph/9912251.

[7] Z. Bern, J.S. Rozowsky and B. Yan, Phys. Lett. B401, 273 (1997) [hep-ph/9702424].

[8] W.L. van Neerven, Nucl. Phys. B268, 453 (1986). Z. Bern, L. Dixon, D.C. Dunbar and D.A. Kosower, Nucl. Phys. B425, 217 (1994) [hep-ph/9403226]; Nucl. Phys. B435, 59 (1995) [hep-ph/9409265];

Z. Bern and A.G. Morgan, Nucl. Phys. B467, 479 (1996) [hep-ph/9511336];

Z. Bern, L. Dixon and D.A. Kosower, Nucl. Phys. B513, 3 (1998) [hep-ph/9708239];

J.S. Rozowsky, hep-ph/9709423.

[9] Z. Bern, L. Dixon, D.C. Dunbar, M. Perelstein and J.S. Rozowsky, Nucl. Phys. B530, 401 (1998) [hep-th/9802162].

[10] Z. Bern, L. Dixon, M. Perelstein and J.S. Rozowsky, Phys. Lett. B444, 273 (1998) [hepth/9809160]; Nucl. Phys. B546, 423 (1999) [hep-th/9811140].

[11] M.T. Grisaru, H.N. Pendleton and P. van Nieuwenhuizen, Phys. Rev. D15, 996 (1977);

M.T. Grisaru and H.N. Pendleton, Nucl. Phys. B124, 81 (1977);

S.J. Parke and T.R. Taylor, Phys. Lett. B157, 81 (1985), err. ibid. B174, 465 (1986).

[12] S. Catani, Phys. Lett. B427, 161 (1998) [hep-ph/9802439].

[13] Z. Kunszt, A. Signer and Z. Trócsányi, Nucl. Phys. B420, 550 (1994) [hep-ph/9401294].

[14] G. 't Hooft and M. Veltman, Nucl. Phys. B44, 189 (1972).

[15] W. Siegel, Phys. Lett. 84B, 193 (1979);

D.M. Capper, D.R. Jones and P. van Nieuwenhuizen, Nucl. Phys. B167, 479 (1980).

[16] Z. Bern and D.A. Kosower, Nucl. Phys. B379, 451 (1992).

[17] Z. Kunszt, A. Signer and Z. Trócsányi, Nucl. Phys. B411, 397 (1994) [hep-ph/9305239].

[18] S. Catani, M.H. Seymour and Z. Trócsányi, Phys. Rev. D55, 6819 (1997) [hep-ph/9610553].

[19] Z. Bern, G. Chalmers, L. Dixon and D.A. Kosower, Phys. Rev. Lett. 72, 2134 (1994) [hep$\mathrm{ph} / 9312333]$

G. Mahlon, Phys. Rev. D49, 4438 (1994) [hep-ph/9312276]. 
[20] V. Del Duca, L. Dixon and F. Maltoni, hep-ph/9910563.

[21] Z. Bern, L. Dixon, D.C. Dunbar and D.A. Kosower, Phys. Lett. B394, 105 (1997) hepth/9611127.

[22] L. Lewin, Dilogarithms and Associated Functions (Macdonald, 1958).

[23] Z. Bern, L. Dixon and D. A. Kosower, Phys. Lett. B302, 299 (1993) [hep-ph/9212308], err. ibid. B318, 649 (1993); Nucl. Phys. B412, 751 (1994) [hep-ph/9306240]. 